\title{
Connectivity-Based Parcellation of Human Cingulate Cortex and Its Relation to Functional Specialization
}

\author{
Matthias Beckmann, ${ }^{1,3}$ Heidi Johansen-Berg, ${ }^{1}$ and Matthew F. S. Rushworth ${ }^{1,2}$ \\ ${ }^{1}$ Centre for Functional MRI of the Brain and 2Department of Experimental Psychology, University of Oxford, Oxford OX1 3UD, United Kingdom, and \\ ${ }^{3}$ Universitätsklinikum “Carl Gustav Carus,” Technische Universität Dresden, 01307 Dresden, Germany
}

\begin{abstract}
Whole-brain neuroimaging studies have demonstrated regional variations in function within human cingulate cortex. At the same time, regional variations in cingulate anatomical connections have been found in animal models. It has, however, been difficult to estimate the relationship between connectivity and function throughout the whole cingulate cortex within the human brain. In this study, magnetic resonance diffusion tractography was used to investigate cingulate probabilistic connectivity in the human brain with two approaches. First, an algorithm was used to search for regional variations in the probabilistic connectivity profiles of all cingulate cortex voxels with the whole of the rest of the brain. Nine subregions with distinctive connectivity profiles were identified. It was possible to characterize several distinct areas in the dorsal cingulate sulcal region. Several distinct regions were also found in subgenual and perigenual cortex. Second, the probabilities of connection between cingulate cortex and 11 predefined target regions of interest were calculated. Cingulate voxels with a high probability of connection with the different targets formed separate clusters within cingulate cortex. Distinct connectivity fingerprints characterized the likelihood of connections between the extracingulate target regions and the nine cingulate subregions. Last, a meta-analysis of 171 functional studies reporting cingulate activation was performed. Seven different cognitive conditions were selected and peak activation coordinates were plotted to create maps of functional localization within the cingulate cortex. Regional functional specialization was found to be related to regional differences in probabilistic anatomical connectivity.
\end{abstract}

Key words: cingulate cortex; DTI; tractography; human; premotor cortex; decision making; emotion

\section{Introduction}

Human cingulate cortex has been implicated in many functions. Regional specialization within cingulate cortex is probably related to its anatomical differentiation into cytoarchitectonically distinct subregions that, in macaque, have distinct connections (Matelli et al., 1991; Van Hoesen et al., 1993; Vogt et al., 1995; Zilles et al., 1995; Strick et al., 1998). The invasive nature of techniques for studying connectional anatomy has meant that little is known directly of the anatomical connections of human cingulate cortex despite the wealth of evidence for functional specialization from neuroimaging studies. The dorsal cingulate sulcus includes at least two and possibly more motor regions that are active during movement (Picard and Strick, 1996; Paus, 2001). Anterior dorsal cingulate sulcus is active when the linking of an action to reinforcement is critical, for example, during error detection or when action selection is guided by the prospect of reward (Holroyd and Coles, 2002; Rushworth et al., 2007). Adjacent regions are implicated in detecting when actions are potentially in conflict (Botvinick et al., 2004). The more ventral cingu-

Received July 15, 2008; revised Nov. 13, 2008; accepted Dec. 2, 2008.

This work was supported by the German National Academic Foundation (M.B.), the Medical Research Council (M.F.S.R.), and the Wellcome Trust (H.J.-B.). We thank Saad Jbabdi, Johannes Klein, and P. M. Matthews for technical assistance and useful discussions.

Correspondence should be addressed to Matthew F. S. Rushworth, Department of Experimental Psychology, University of 0xford, 0xford 0X1 3UD, UK. E-mail: matthew.rushworth@psy.ox.ac.uk.

DOI:10.1523/JNEUROSCI.3328-08.2009

Copyright $\odot 2009$ Society for Neuroscience $\quad$ 0270-6474/09/291175-16\$15.00/0 late gyrus is active when subjects experience pain (Vogt, 2005) and during autonomic arousal (Critchley et al., 2000). Both ventral cingulate and anterior dorsomedial paracingulate regions are active during emotion and social interaction (Vogt, 2005; Amodio and Frith, 2006). Posterior and retrosplenial areas are implicated in memory (Maguire, 2001). Given its importance in diseases such as Alzheimer's disease, depression, schizophrenia, and obsessive compulsive disorder (Rosenberg et al., 2004; Gotlib et al., 2005; Kubicki et al., 2005; Naggara et al., 2006), it is desirable to identify human cingulate subregions on the basis of both anatomical connectivity and function in vivo.

Here, two complementary magnetic resonance imaging (MRI)-based strategies were used to identify regional differences in anatomical connectivity in human cingulate cortex by means of probabilistic diffusion tractography. First, we examined the probabilistic connectivity profiles of all cingulate cortex voxels to detect inter-regional differences. Such differences define areal divisions in premotor and ventral prefrontal cortex (JohansenBerg et al., 2004; Rushworth et al., 2006; Anwander et al., 2007; Klein et al., 2007; Tomassini et al., 2007). This "blind," a priori approach makes no assumptions about the identities of the interconnecting regions driving the parcellation of the area under investigation. By calculating cross-correlations between the connectivity profiles of all cingulate voxels and reordering them by applying $k$-means clustering (Klein et al., 2007), we characterized a total of nine separate cingulate areas.

Second, a complementary a posteriori approach focused on 11 
brain areas known to interconnect with cingulate cortex in other primate species. Each of these areas were used as targets for guided probabilistic tractography from the cingulate seed masks (CSMs) to estimate their probability of interconnection.

We created connectivity fingerprints (Passingham et al., 2002; Tomassini et al., 2007) to combine the results from both approaches and to visualize the likely anatomical connections of each identified cingulate cluster. In addition, to assess the relationship between anatomical connectivity and function, we also compared our results with a meta-analysis of functional neuroimaging studies reporting cingulate activation in eight different task conditions.

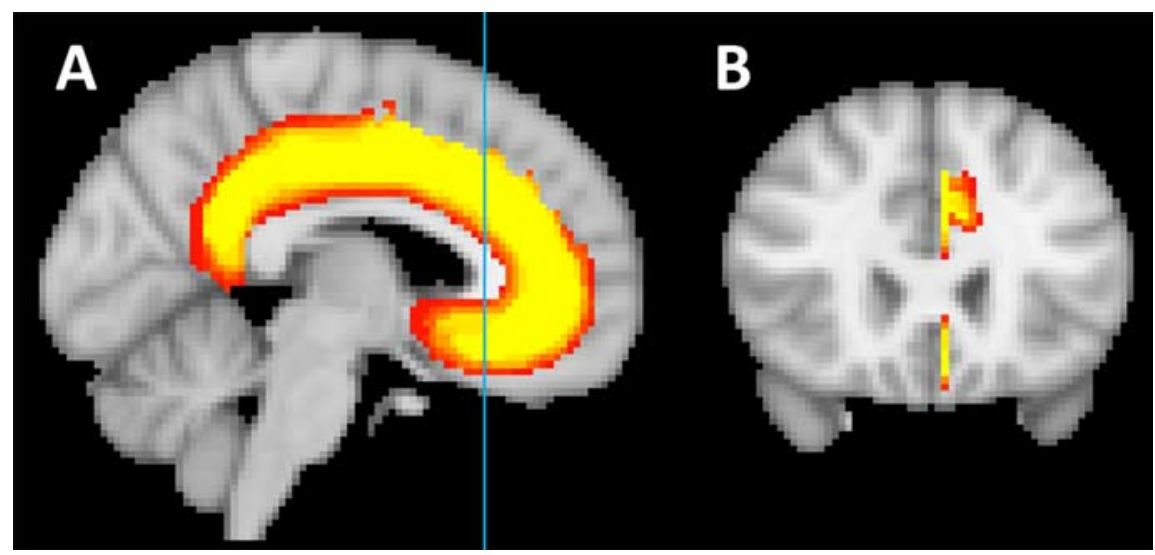

Figure 1. $\quad \boldsymbol{A}$, The group average whole CSM superimposed on the group average structural MRI scan in MNI space on a sagittal section $(x=-4)$. $\boldsymbol{B}$, Coronal section at $y=24$ (as marked by the light blue line in $\boldsymbol{A}$ ), showing the lateral extension of the mask into paracingulate and sulcal areas.

\section{Materials and Methods}

In this study, diffusion tractography was used in two complementary but distinct ways to characterize human cingulate probabilistic connectivity. First, cingulate cortex was parcellated into distinct subregions on the basis of regional differences in the probabilistic connectivity profiles of each cingulate voxel. This approach made no a priori assumptions about the identity of the extracingulate regions that would drive the regional differences in connectivity profile within the cingulate cortex. Second, connections were estimated between cingulate cortex and 11 target regions known to be interconnected in another primate species: the macaque. This part of the study therefore constituted a complementary a posteriori approach. As a third part of this study, we performed a meta-analysis of previous reports of cingulate activation in various behavioral conditions and examined the interrelationship of functional activations to estimated anatomical connectivity.

\section{Diffusion tractography}

\section{Subjects, data acquisition, and analysis}

Diffusion-weighted (three repeats of 60 diffusion directions; $b=1000$ $\mathrm{s} / \mathrm{mm}^{2} ; 2 \times 2 \times 2 \mathrm{~mm}$ voxels) and T1-weighted images were acquired in 11 healthy volunteers (seven male, four female; aged $20-36$ years) on a 1.5 T Siemens Sonata MRI scanner in accordance with local ethical approval. Data were analyzed using tools from the Oxford University Centre for Functional MRI of the Brain (FMRIB) software library (http:// www.fmrib.ox.ac.uk/fsl). Motion and eddy current correction as well as image averaging were performed on the diffusion data. Before analysis, the structural volumes were registered to Montreal Neurological Institute (MNI) standard space using the linear registration tool FLIRT by FMRIB.

Diffusion modeling applied a probabilistic diffusion model (Behrens et al., 2003), modified to allow for estimates of multiple fiber directions (Behrens et al., 2007b). For each subject, probabilistic tractography was run from voxels within the whole cingulate cortex seed area to assess connectivity with every brain voxel. The approach draws a sample from each fiber orientation distribution at the current voxel and chooses the sample closest to the orientation of its previous step. For each subject, we initiated 1000 samples from the connectivity distribution from each seed voxel. The connection probability between a cingulate voxel and another voxel in the brain is defined as the sum of sample fiber lengths connecting these two voxels. This value corresponds approximately to the connection probability (i.e., the total number of samples connecting the two regions) multiplied by the average connection path length. It corrects for the fact that distant regions appear less connected than close regions when using the probability alone and is appropriate for parcellationbased studies in which distant connections are the principal focus and quantitative probability values are not required. The algorithm was limited to estimating two fiber orientations at each voxel because of the $b$ value and number of gradient orientations in the diffusion data (Behrens et al., 2007b).
Definition of cingulate seed masks

Masks were manually drawn on a single left-hemisphere sagittal slice $(x=-4)$ on each individual's T1-weighted anatomical scan, after registration to standard (MNI 152) space, to include all tissue commonly assigned to the cingulate cortex (Fig. 1). We refer subsequently to this mask as the whole CSM. This plane was appropriate because it covered the entire cingulate gyrus in all subjects. However, to include the cingulate and, if applicable, the paracingulate sulci, the masks were chosen to extend laterally on to neighboring slices in these particular regions. In practice, this meant that, in the vicinity of the cingulate and paracingulate sulci, the mask extended as far as $x=-10$ in some subjects. Mask boundaries were defined as follows. In the supracallosal portion of the cingulate cortex, the ventral boundary of the CSM was the corpus callosum. The rostral sulcus was taken as the ventral boundary of the cingulate cortex in which it lay anterior or ventral to the genu of the corpus callosum (Paus et al., 1996a,b). When present, the fundus of the paracingulate sulcus was taken as the anterior and dorsal boundary of the CSM. The dorsal bank of the cingulate sulcus was taken as the dorsal limit of the CSM at more posterior levels and also at anterior positions when the paracingulate sulcus was absent (Vogt et al., 1995; Paus et al., 1996a,b). Whenever the cingulate sulcus appeared broken, the dorsal limit of the mask followed an imaginary line between adjacent parts of the cingulate sulcus. The subparietal sulcus was the dorsal boundary of the CSM in the most posterior part of the cingulate cortex posterior to the marginal sulcus. In this region, the dorsal boundary of the mask was an imaginary line drawn between the posterior limit of cingulate sulcus and the anterior limit of the subparietal sulcus when these two sulci were not directly joined with one another. The posterior boundary of the mask was an imaginary line drawn along the shortest route between the posterior boundary of the subparietal sulcus and the nearest point on the corpus callosum. Care was taken to ensure that the masks stayed clear of the corpus callosum itself as well as of the cingulum bundle. In both approaches, the cingulate masks then served as seeds for probabilistic diffusion tractography.

\section{Connectivity-based parcellation}

The blind connectivity-based parcellation of human cingulate cortex was performed iteratively. Connections were estimated between all voxels in the CSM and all remaining voxels in the rest of the brain (which had been stored at a lower resolution with a voxel size of $5 \times 5 \times 5 \mathrm{~mm}$ ). After an initial parcellation step had subdivided the cingulate into subregions, these subregions were then used as seed masks for additional iterations of the parcellation procedure.

In more detail, the following steps were used. First, probabilistic tractography was performed and values were obtained for all voxels in the CSM and their probability of connection to all remaining voxels in the brain. Results were then stored in a two-dimensional matrix of cingulate cortex seed voxels by extracingulate brain voxels. From this initial, native connectivity matrix, a cross-correlation matrix was calculated that rep- 


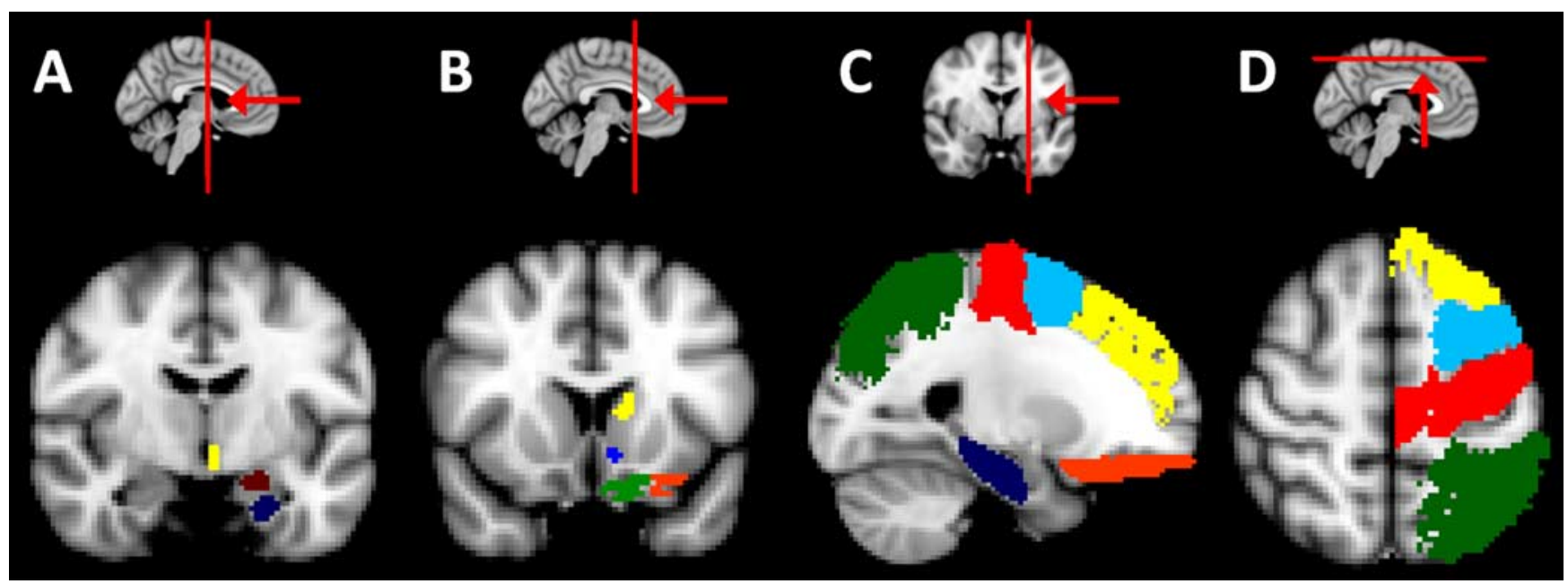

Figure 2. Group average extracingulate target masks superimposed on the MNI 152T1 standard brain. A, A coronal section $(y=-8)$ showing parts of the group average hypothalamus (yellow), amygdalae (dark red), and hippocampus (dark blue) target masks. B, A coronal section $(y=14$ ) showing the group average dorsal striatum (yellow), ventral striatum (light blue), medial (green), and lateral (orange) orbitofrontal cortex target masks. C, A sagittal section $(x=-18)$ showing the group average hippocampus (dark blue), lateral orbitofrontal (orange), dorsal prefrontal (yellow), premotor (light blue), precentral (red), and parietal (green) cortex target masks. D, An axial section $(z=56)$ showing the group average dorsal prefrontal (yellow), premotor (light blue), precentral (red), and parietal (green) cortex target masks.

resented the correlation between connectivity profiles of cingulate seed voxels. The cross-correlation matrix was then reordered using $k$-means clustering (Klein et al., 2007; Tomassini et al., 2007), so that voxels were more likely to be grouped together the more similar their connectivity profiles. In this step, no spatial constraint was applied when identifying clusters, and the grouping of voxels was entirely dependent on diffusion information. The number of component clusters was, however, chosen by the experimenter. It was set to be the highest number that produced a consistent, comparable, and spatially similar pattern of clustering across subjects (see Fig. 3). Some of the component clusters identified in this manner were then subject to an additional iteration of analysis.

Initially, we found evidence for five distinct clusters. This is discussed in detail in Results. In brief, the largest cluster was a discontinuous region that included a large part of posterior cingulate cortex and a large part of anterior cingulate cortex that were separated from one another by the other four clusters. A second parcellation iteration was therefore performed to further examine the connections of the large anterior and posterior cingulate cortex components separately. Additional analysis iterations did not lead to parcellations that were reliably similar across subjects.

\section{Probabilistic interconnections between cingulate cortex and 11 target regions}

In the second part of the study, identical cingulate cortex seed masks were used as in the initial stage of the parcellation analysis. On the basis of the known connections of the macaque cingulate cortex, we also defined 11 target regions of interest elsewhere in the brain as multislice threedimensional masks (Fig. 2). Diffusion tractography was then used to estimate the probability of interconnection between voxels in the CSM and each of the other extracingulate regions within the same hemisphere.

For each subject, we drew 5000 samples from the connectivity distribution from each seed voxel in the CSM and computed the probability of connection with each of the 11 target masks. A connection probability value was recorded for every CSM voxel in relation to every target mask.

For each subject, the median probability of connection across voxels between each of the nine cingulate subregions identified within the CSM and each extracingulate target region was computed. Subsequently, median probability of connection across subjects was calculated. We then normalized these values by dividing by the total sum of probability of connection between a given extracingulate region and all nine cingulate regions so that these values represent the connection probability of each cluster as a proportion of total cingulate connections for a given extracingulate region (Passingham et al., 2002; Croxson et al., 2005). Normal- ized connection probabilities were then used to generate connectivity fingerprints.

The connectivity maps of cingulate voxels classified according to probability of connection to target regions for each individual subject, thresholded at the 85th percentile of the highest connection probability value for each target, were then binarized. Overlays of the binarized results generated in all 11 subjects' brain volumes were then created to visualize the reproducibility of the results across subjects. Such a method of visualizing the results entails a very strict requirement: that the precise same voxels, and not merely adjacent voxels within the same mask, be strongly implicated in a connection in several subjects. To provide a clear and comprehensive summary of connections, two steps were therefore taken. First, group connectivity maps were thresholded at two subjects so that colored voxels indicate voxels that were strongly implicated in a connection with a given area in at least two subjects. In addition, the yellow voxels in the maps indicate that a connection was common to eight or more subjects (see Fig. 5). Second, the results were also summarized as connectivity fingerprints, which are independent of the thresholding applied to the spatial maps. The fingerprints therefore give a threshold-free indication of the average strength (across all subjects) of connection between each cingulate cluster and each target region.

\section{Definition of target masks}

With the exception of the orbitofrontal cortex masks, the extracingulate cortical masks were adapted from studies [premotor cortex (Tomassini et al., 2007), parietal cortex (Rushworth et al., 2006), precentral gyrus (Smith et al., 2004), and dorsal prefrontal cortex (Croxson et al., 2005)] that had defined these regions previously. The masks used to define the subcortical target regions and the orbitofrontal cortex, however, were drawn specifically for this study. All subcortical masks were delineated on each individual's structural images and only then registered to standard space.

Amygdala and hippocampus. The probability of connections between cingulate and amygdala was assessed because, in the monkey, the amygdala is interconnected with anterior cingulate cortex (Porrino et al., 1981; Amaral and Price, 1984; Carmichael and Price, 1995; Ghashghaei et al., 2007; Morecraft et al., 2007).

The hippocampal formation, particularly the subiculum, is interconnected with the ventromedial anterior cingulate and with retrosplenial cingulate cortex (Vogt et al., 1987; Carmichael and Price, 1995; Barbas et al., 1999; Parvizi et al., 2006). Care was taken to include the subiculum within the hippocampal mask. Because of the proximity of the white matter subjacent to the subiculum and the white matter subjacent to the 
entorhinal cortex, there is a possibility that some entorhinal connections were also included. The parahippocampal gyrus was not included in the mask, and its position lateral to the collateral sulcus (Pruessner et al., 2002) means that it is unlikely that hippocampal connectivity estimates are confounded with parahippocampal connectivity estimates.

The amygdala mask was drawn onto six consecutive coronal slices; in this way, the mask extended throughout the full anteroposterior extent of the amygdala. For the creation of the hippocampus mask, we also used coronal structural sections. The hippocampal mask extended from the head of the hippocampus anteriorly to the tail of the hippocampus at the level of the last coronal slice on which the mesencephalon was still visible. Care was taken to ensure that amygdala and hippocampus masks did not overlap, and, consequently, tissue at the boundary that could not be confidently assigned to either the amygdala or hippocampus was excluded from both masks.

Ventral and dorsal striatum. In the monkey, much of the striatum is interconnected with the cingulate cortex. At a first approximation, ventral striatum is more strongly interconnected with ventral cingulate cortex, whereas more dorsal cingulate cortex, including the cingulate motor areas (CMAs) in the supracallosal cingulate sulcus, is more strongly connected with the dorsal striatum (Kunishio and Haber, 1994). The connections of the tissue in the cingulate motor area and immediately anterior, however, range over a wide extent of the anterior striatum, including both the caudate and putamen (Takada et al., 2001; Haber et al., 2006).

Although there are some projections from posterior cingulate to ventral striatum, the strongest projections are from ventral anterior cingulate cortex (Kunishio and Haber, 1994; Haber et al., 1995; Parvizi et al., 2006). It is important to note, however, that even ventral anterior cingulate cortex connections within the striatum are not limited to just its ventral part but extend to include dorsal caudate regions, too (Haber et al., 2006).

In the present study, the ventral striatum was defined in coronal slices of the structural T1-weighted images. We only included gray matter that could be assigned unambiguously to the ventral striatum. The dorsal striatum mask solely included parts of the caudate nucleus and the putamen that were in, or dorsal to, the first axial MRI slice to include both the genu and the splenium of the corpus callosum. This meant that an ambiguous intermediate region that was not easily assigned to either ventral or dorsal striatum was not included in either mask. A similar procedure has been used previously by Croxson et al. (2005).

Hypothalamus. The ventromedial and anterior cingulate cortex and adjacent medial orbitofrontal cortex are distinguished from other prefrontal regions by the density of their projections to the hypothalamus (Ongür et al., 1998; Freedman et al., 2000). Although the connections of subgenual cingulate cortex with the hypothalamus have received the most attention, it is clear that, in the monkey, the most ventral tier of supracallosal cingulate cortex also projects to hypothalamus (Ongür et al., 2003). Posterior cingulate cortex has some connections with adjacent regions of the zona incerta (Parvizi et al., 2006) that might also be detected with diffusion-weighted imaging (DWI) tractography.

The hypothalamus mask was drawn on $\mathrm{T} 1$ coronal sections and included all hypothalamic gray matter from $\sim 2 \mathrm{~mm}$ posterior to the coronal level of the optic chiasm to a coronal level $\sim 2 \mathrm{~mm}$ anterior to the coronal level of the subthalamic nucleus. The anterior and posterior borders of the hypothalamus mask were therefore at approximately $y=$ -2 and $y=-10$, respectively.

Parietal cortex. In the monkey, posterior cingulate cortex is interconnected with an extended region of both superior and inferior posterior lateral parietal cortex (Jones and Powell, 1970; Vogt and Pandya, 1987; Cavada and Goldman-Rakic, 1989; Morecraft et al., 2004). It is interesting to note that the posterior cingulate regions that are interconnected with parietal cortex primarily fail to overlap with the anterior cingulate regions in which interconnections with amygdala are prominent (Van Hoesen et al., 1993). It has been suggested that, within cingulate cortex, there is a fundamental dichotomy between the functions of anterior and posterior regions, with posterior regions being more concerned with spatial representation and orientation and with memory (Baleydier and Mauguiere, 1980; Vogt et al., 1992). The cingulate region most strongly interconnected with the parietal cortex corresponds approximately to the region commonly referred to as posterior cingulate cortex.

The connections of the human parietal cortex with the cingulate cortex were, therefore, also estimated. The connections with a large region of parietal cortex were estimated because of the distributed nature of parietal-posterior connections and because aspects of the correspondence between human and macaque parietal cortex remain contentious (Rushworth et al., 2006; Husain and Nachev, 2007). The mask used was similar to the parietal cortex mask examined in a recent study of the subcortical connections of the parietal cortex (Rushworth et al., 2006). The parietal cortex masks drawn for individual subjects in that study were combined in MNI space. The resulting combination image was thresholded so that it included voxels identified as parietal cortex in at least half of the subjects; the resulting image therefore contained some discontinuous voxels.

Orbitofrontal cortex. In the macaque, the anterior cingulate cortex is connected with the orbitofrontal cortex (Morecraft and Van Hoesen, 1993; Cavada et al., 2000). The connections between the medial orbitofrontal cortex and the ventromedial anterior cingulate cortex are particularly strong, and Price and Carmichael have argued that together these areas comprise a medial frontal network. It is argued that this network can be distinguished from an "orbitofrontal" network that is centered on lateral orbitofrontal cortex (Carmichael and Price, 1996). The ventromedial and lateral orbitofrontal cortical networks are only sparsely interconnected with one another (Carmichael and Price, 1996).

We used a combination of axial and sagittal views to create the orbitofrontal cortex masks. Both were drawn in the left hemisphere and across eight consecutive axial slices, ensuring that there was no overlap with the neighboring cingulate cortex mask. The drawing of the masks was guided by the descriptions of orbitofrontal sulcal anatomy made by Chiavaras and colleagues (Chiavaras and Petrides, 2000; Chiavaras et al., 2001), and it was similar to the masks used by Croxson et al. (2005).

In the present study, two orbitofrontal masks were used. The lateral orbitofrontal mask extended from the horizontal ramus of the Sylvian fissure on the lateral surface to the medial orbital sulcus on the orbital surface. The anterior boundary was a line drawn between the anterior tips of the horizontal ramus of the Sylvian fissure, the lateral orbitofrontal sulcus, and the medial orbitofrontal sulcus. The posterior boundary was a line drawn between the posterior boundaries of the same sulci. The medial orbitofrontal mask extended from the medial bank of the medial orbital sulcus to the rostral sulcus on the medial surface. The anterior boundary was a line drawn between the anterior tips of the medial orbital sulcus and the rostral sulcus, and the posterior boundary was a line drawn between the posterior boundaries of the same two sulci.

Premotor cortex. In the macaque, the supracallosal cingulate sulcus contains at least two and possibly three motor regions in which microstimulation leads to limb movement and which are connected to the ventral horn of the spinal cord (Mitz and Wise, 1987; Hutchins et al., 1988; Dum and Strick, 1991, 1993, 1996; Luppino et al., 1991, 1994; Matelli et al., 1991; Shima et al., 1991; Morecraft and Van Hoesen, 1992; He et al., 1995; Morecraft et al., 1996). In the macaque, the most anterior CMA is situated anterior to the coronal plane of the bow of the arcuate sulcus. It is often referred to as the rostral CMA (CMAr). It has been argued that the caudal CMA can be subdivided into dorsal and ventral $(\mathrm{CAMv})$ components. In the monkey, all of the cingulate motor areas are interconnected with dorsal and ventral divisions of lateral premotor cortex (Barbas and Pandya, 1987; Luppino et al., 1998, 2003; Hatanaka et al., 2003; Takada et al., 2004). There is little evidence for connections between the cingulate gyrus, ventral and posterior to the CMAs, and the lateral premotor cortex.

The probability of connection between the cingulate cortex mask and the lateral premotor cortex was therefore investigated. The posterior border was approximately two-thirds of the way across the precentral gyrus (Alkadhi et al., 2002a,b), the inferior border was the ventral limit (Zhang et al., 2001) of the precentral gyrus, and the medial border was $\sim 5 \mathrm{~mm}$ from the medial surface of the brain. The anterior border was located $\sim 5 \mathrm{~mm}$ in front of the precentral sulcus (Tomassini et al., 2007) because it has been proposed that a part of human ventral premotor cortex might lie anterior to the inferior precentral sulcus (Geyer et al., 2000). 
Precentral cortex. In the macaque, the CMAs are also interconnected with the primary motor cortex, located immediately posterior to the lateral premotor cortex. Connections between the CMAr and primary motor cortex are, however, weak compared with those between caudal CMAs and primary motor cortex (Wang et al., 2001; Hatanaka et al., 2003; Wang et al., 2004). The probability of connections between a precentral cortex region posterior to the premotor cortex was therefore also examined. The precentral gyrus masks were derived from the atlas incorporated in the latest version of FSLview (Johansen-Berg et al., 2004) (http://www.cma.mgh.harvard.edu/manuals/).

Dorsal prefrontal cortex. In the macaque, dorsal prefrontal cortex is interconnected with much of cingulate cortex (Bates and GoldmanRakic, 1993; Lu et al., 1994). The region of interconnection includes, on the one hand, much of dorsal prefrontal cortex and, on the other hand, tissue in the dorsal supracallosal cingulate cortex, including that in or adjacent to the CMAs, particularly CMAr and CMAv, but extends to include more anterior cingulate sulcus and parts of the cingulate gyrus. The dorsal prefrontal mask was based on one used previously (Croxson et al., 2005) and extended from the inferior frontal sulcus on the lateral surface to $\sim 1 \mathrm{~cm}$ above the paracingulate sulcus (or, when this was absent, cingulate sulcus) on the medial surface. The mask did not include the frontal pole. Care was taken to ensure that there was no overlap between the mask and the adjacent cingulate cortex mask.

\section{Meta-analysis of functional studies}

The following conditions were considered for the meta-analysis: pain, motor function, emotion, error, conflict, reward, and memory. The PubMed database (http://www.ncbi.nlm.nih.gov/entrez/) was searched for functional MRI (fMRI) and positron emission tomography studies, published between January 1, 2002 and April 15, 2007, containing both the term "cingulate cortex" or "medial prefrontal cortex" and one of the conditions specified above. The search was limited to the following journals: Neuroimage, The Journal of Neuroscience, Cerebral Cortex, The Proceedings of the National Academy of Sciences of the U S A, Brain Research, Neuron, Neuropsychologia, The Journal of Cognitive Neuroscience, Brain, Experimental Brain Research, The European Journal of Neuroscience, Trends in Cognitive Sciences, The Journal of Comparative Neurology, Nature Neuroscience, Nature Reviews Neuroscience, Human Brain Mapping, and Biological Psychiatry.

We retrieved the peak activation coordinates of a total of 171 original publications. To visualize the activation centers, all study coordinates referred to the MNI (Collins et al., 1994) template or were converted to MNI space (http://wwwneuro03.uni-muenster.de/ger/t2tconv/conv3d.html) when only Talairach coordinates were provided. The coordinates were then mapped onto the MNI 152 standard template, one voxel per study (in cases in which more than one cingulate peak was reported, only the set of coordinates with the highest degree of significance was included). To allow for comparison with the tractography results, all of these activation sites were eventually projected onto one single sagittal plane $(x=$ $-4)$.

\section{Results}

\section{Connectivity-based parcellation}

The first iteration of the parcellation of the whole cingulate seed mask identified five clusters with substantially distinct connectivity profiles in both subjects lacking a well defined paracingulate sulcus and subjects with an identifiable paracingulate sulcus. Four of the clusters were found in the midcingulate region. Most of the voxels assigned to each of these four clusters were spatially adjacent to one another. The rest of the cingulate comprised one large discontinuous cluster with an anterior and a posterior component. As explained below, the anterior and posterior components of this large cluster resembled one another in that they shared a high probability of interconnection with hippocampus and hypothalamus. Although the investigation of intracingulate connections is beyond the scope of the current report, it should also be noted that these regions are known to be directly inter- connected with one another in the macaque (Van Hoesen et al., 1993; Parvizi et al., 2006). Van Hoesen et al. (1993) reviewed a series of 14 cases in which both small and large anterograde and retrograde tracer injections were made in macaques to demonstrate the general point that anterior cingulate cortex and posterior cingulate cortex are more strongly interconnected with one another than they are with midcingulate cortex. These anterior and posterior components were separated and submitted to independent additional parcellation iterations. In the second parcellation iteration, we identified three subclusters in the anterior cingulate cortex and two in the posterior cingulate cortex in both subjects lacking a well defined paracingulate sulcus (Fig. $3 A, C$ ) and subjects with an identifiable paracingulate sulcus (Fig. $3 B, D$, top).

In this way, a total of nine distinct cingulate subregions were identified by iteratively parcellating the whole of the cingulate cortex. Three anterior, four midcingulate, and two posterior cingulate clusters could be distinguished in both all three subjects without a clearly defined paracingulate sulcus (Fig. $3 A, C$ ) and all eight subjects with an identifiable paracingulate sulcus (Fig. $3 B, D)$. The results were therefore consistent across all 11 individuals (Fig. $3 E, F$ ). More fine-grained parcellations did not produce consistent results across subjects and were therefore not considered further.

A number of the clusters identified by the parcellation procedure can be related to areas previously identified in cytoarchitectonic and anatomical investigations of the cingulate cortex and adjacent medial frontal cortex (Fig. 4). Cluster 1 overlaps with the subcallosal anterior cingulate cortex region identified in a diffusion-weighted imaging and tractography study that focused exclusively on the tissue anterior and ventral to the genu of the corpus callosum (Johansen-Berg et al., 2008) (Fig. 4). Cytoarchitectonic and comparative studies of the human cingulate cortex have identified area 25 in a similar location (Petrides and Pandya, 1994; Vogt et al., 1995; Palomero-Gallagher et al., 2008). According to one group of researchers, this region may be further divisible into areas 25 and a prelimbic part of area $32,32 \mathrm{pl}$, or $32 \mathrm{~m}$ (Ongür and Price, 2000; Ongür et al., 2003).

Cluster 7 was situated dorsal to the corpus callosum and, in most subjects, clearly extended anteriorly into pregenual cingulate gyrus. Because the pregenual part of the cluster 7 of the different subjects did not overlap completely after registration into the standard MNI space, cluster 7 appears patchy anterior to the corpus callosum (Figs. 3, 4). Cluster 7 occupies an approximately similar position to area 24 of the human cingulate gyrus (Petrides and Pandya, 1994; Vogt et al., 1995; Ongür et al., 2003). The pregenual part of cluster 7 corresponds to the pregenual region identified by Johansen-Berg et al. (2008) in their study of the perigenual cingulate cortex (Fig. $4 A$ ). The between-subject overlap in the pregenual region may be more apparent in the study by Johansen-Berg et al. because of the larger number of participating subjects. In comparing cluster 7 with the findings reported by Ongür et al. and Johansen-Berg et al., it is important to remember that these previous studies only investigated a limited region of anterior cingulate and medial frontal cortex, and little of the supracallosal region, in which the main part of cluster 7 was found in the present study, was included in their analyses.

Cluster 2 was situated dorsal to the rostral sulcus anterior and ventral to the genu of the corpus callosum. This region was included in the analysis because it has been treated as anterior cingulate cortex and referred to as area 32 by most investigators (Brodmann, 1909; Petrides and Pandya, 1994; PalomeroGallagher et al., 2008). Palomero-Gallagher and colleagues have 
reported that area 32 is a continuous band of dysgranular tissue dorsal, anterior, and ventral to the rostral corpus callosum and occupying regions similar to our clusters 2 and 3. Palomero-Gallagher and colleagues, however, emphasize a number of differences between more dorsal and ventral parts of area 32 and subdivide them into subgenual and pregenual areas s32 and $\mathrm{p} 32$. The current results suggest that the cytoarchitectural differences between s 32 and p32 are mirrored by differences in connectivity. Another group of researchers has also distinguished between cortex in approximately similar regions to clusters 2 and 3 (Ongür et al., 2003). Ongür and colleagues, however, argued that tissue in the vicinity of cluster 2 might correspond to the medial subdivision of area 10 (Fig. $4 D, 10 \mathrm{~m}$ ). Other studies have also suggested that a part of area 10 extends posteriorly into medial frontal cortex between the ventral anterior cingulate cortex and the medial orbitofrontal cortex in other primate species (Carmichael and Price, 1994; Vogt et al., 2005).

Cluster 3 was also situated in the anterior cingulate cortex dorsal to cluster 1, 2, and 7 and in the vicinity of the probable location of the paracingulate sulcus (Paus et al., 1996a,b). The location of cluster 3 resembles that of dorsal area 32 (Brodmann, 1909; Petrides and Pandya, 1994) or more specifically $32 \mathrm{p}$ (PalomeroGallagher et al., 2008) or 32ac/32h (Ongür and Price, 2000; Ongür et al., 2003). Once again, when comparing cluster 3 with the results of Ongür, Price, and colleagues, it is important to remember that the previous study did not investigate the tissue assigned to the more posterior part of the cluster 3 mask in the present study.

Clusters 4-6 were situated in the dorsal midcingulate sulcus and supracallosal sulcus, and their positions were reminiscent of regions within areas 24c and 23c (Vogt et al., 1995; Paus, 2001). Clusters 8 and 9 in the posterior cingulate cortex resembled area 23a/b and area 31 (Vogt et al., 1995) in their positions (Fig. 3).

\section{Probabilistic interconnections between cingulate cortex and 11 target regions \\ Amygdala}

Voxels with the highest probability of connection with amygdala were located in the subgenual and pregenual anterior cingulate cortex. The highest connection probabilities were for clusters 1, 2, and 7 , with the highest relative contribution for subgenual cluster 1 (Fig. 5A, Table 1).

\section{Hippocampus}

Across subjects, cingulate voxels with the highest probability of connection with hippocampus were detected in the most anterior the MNI standard brain.
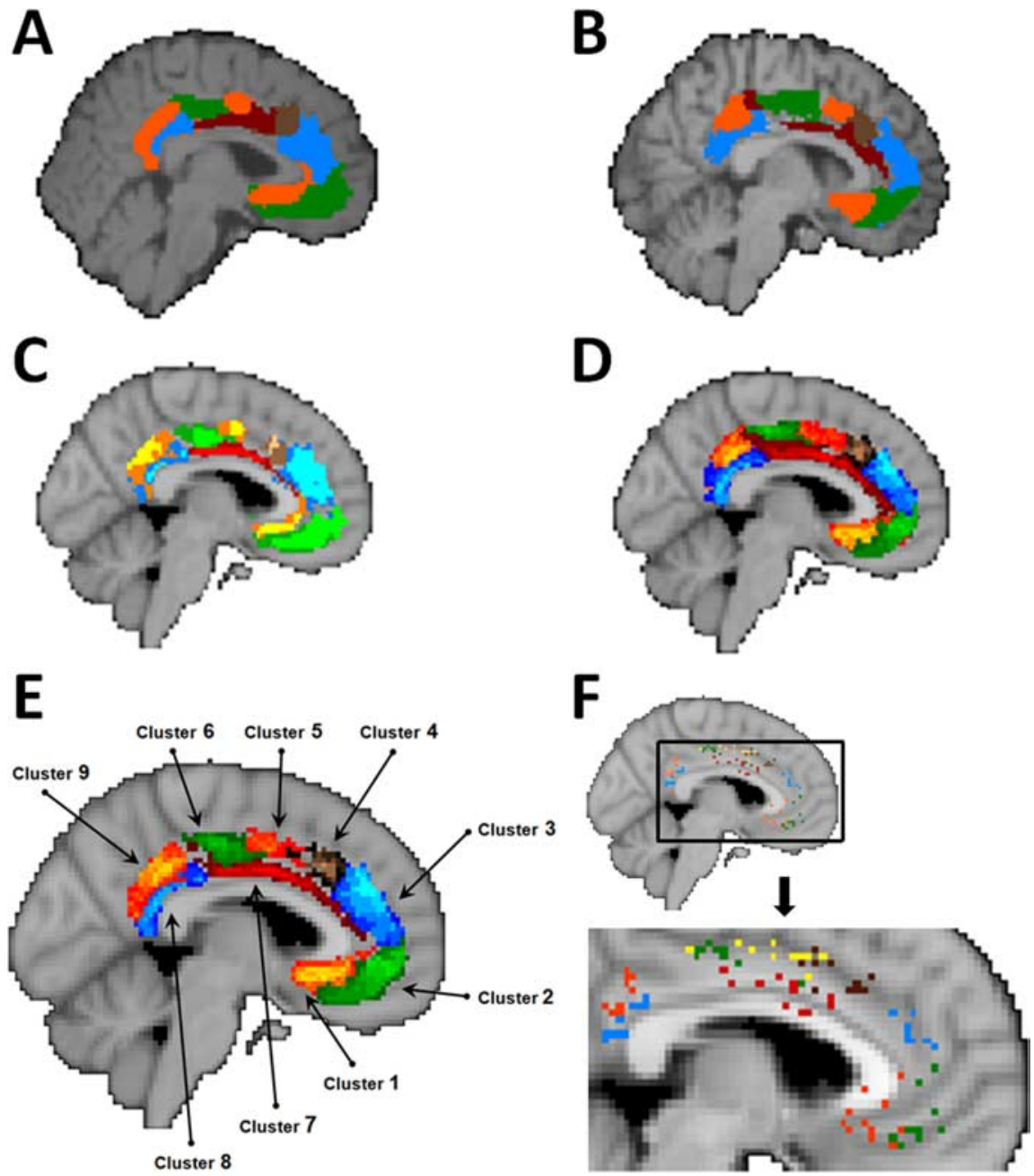

Figure 3. Connectivity-based parcellation of human cingulate cortex. The parcellation analysis resulted in the reliable detection of nine clusters in similar positions in all subjects. The position of the nine clusters in an example subject who lacked a clearly defined paracingulate sulcus $(\boldsymbol{A})$ and in a subject with an identifiable paracingulate sulcus $(\boldsymbol{B})$. Average maps were constructed for the group of subjects $(n=3)$ without clearly identifiable paracingulate sulci $(\boldsymbol{C})$ and the group $(n=8)$ with identifiable paracingulate sulci $(\boldsymbol{D})$. Clusters are identified on group maps wherever there is an overlap in their positions in more than two subjects (for the group of 3 subjects without paracingulate sulci; $\boldsymbol{C}$ ) or more than five subjects (for the group of 8 subjects with paracingulate sulci; $\boldsymbol{D})$. Clusters can be seen in approximately similar positions in the two groups of subjects. $\boldsymbol{E}$, The average positions of the clusters ( 6 subject threshold overlap) for the entire group of 11 subjects is also shown. $\boldsymbol{F}$, The variability in cluster position across individuals can be visualized by plotting the center of gravity of each cluster in each individual on the same image. Points of the same color refer to the centers of gravity of a given cluster in each subject. Occasionally, the center of gravity of a given cluster was the same in two subjects, and only a single point is plotted to represent both subjects. The centers of gravity of different clusters never overlapped in different subjects. Some of the centers of gravity were placed on the corpus callosum, although the clusters themselves did not overlap with the corpus callosum. All MRI scans are sagittal sections $[x=-4$, MNI coordinate system (Collins et al., 1994)]. Individual subject data are shown on the same individuals' structural MRI scans. All group data are shown on

and the most posterior parts of the cingulate cortex in subgenual and retrosplenial areas, but there was also some evidence for connections in the most ventral supracallosal cingulate gyrus (Fig. 5B, Table 1). Additional analysis confirmed that the highest probability of connection with the hippocampus was with clusters 1,8 , and 7 .

\section{Ventral striatum}

The region with a high probability of interconnection with the ventral striatum resembled that with a high probability of connection with the amygdala (Fig. 5A). The highest probability of connection was found for cluster 1 , but there was also evidence for interconnection with clusters 2, 3, and 7 (Fig. 5C, Table 1). 

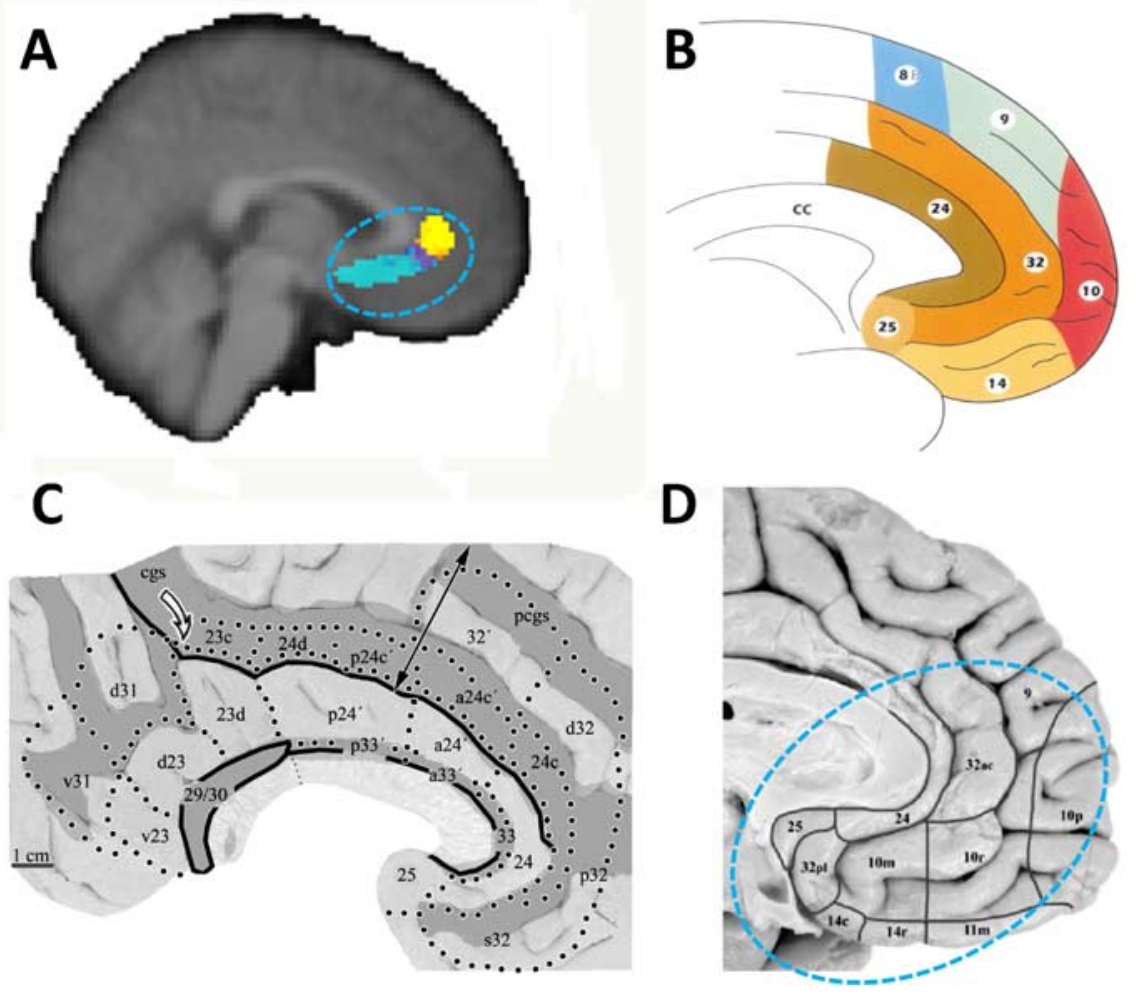

Figure 4. The parcellation clusters resemble regions identified in previous studies. $\boldsymbol{A}$, Cluster 1 occupies a similar position to the subcallosal cingulate region identified by Johansen-Berg et al. (2007). Johansen-Berg and colleagues performed a diffusionweighted imaging tractography parcellation analysis of the region targeted in deep brain stimulation for depression (area colored blue or yellow within the oval). They identified two component clusters (shown in blue and in yellow), one of which (yellow) corresponds in location to cluster 1 in the current study. Cluster 1 also occupies a similar position to area 25 as identified in previous cytoarchitectonic analyses of the human brain conducted by Petrides and Pandya (1994), figure adapted by mirroring to match brain orientation $(\boldsymbol{B})$, and Vogt (2008), figure adapted by mirroring to match brain orientation (C). Note that, in this recent diagram, the label 24 is assigned to tissue sometimes divided into subareas $24 \mathrm{a}$ and $24 \mathrm{~b}$, and the labels $23 \mathrm{~d}, 23 \mathrm{v}$, and v23 are assigned to tissue sometimes divided into 23 a and 23b. D, According to some researchers (Ongur et al., 2003), this region may be further subdivided into areas 25 and 32pl. The positions of clusters 2 and 3 resemble those of the medial extension of area 10,10m, and 32ac (D). Vogt et al. (2005) have also identified area $10 \mathrm{~m}$ in a similar position ventral to cingulate cortex and dorsal to orbitofrontal cortex in the macaque (data not shown). It is important to note that Ongur and colleagues only analyzed the region outlined by the light blue dotted line, and so they did not report on the posterior extension of area 32ac. The position of clusters $4-6$ resemble those of cingulate sulcus regions $24 \mathrm{c}$ and $23 \mathrm{c}(\boldsymbol{C})$. The position of cluster 7 resembles that of $24 \mathrm{a} / \mathrm{b}(\boldsymbol{B}-\boldsymbol{D})$. The positions of clusters 8 and 9 resemble those of areas $23 \mathrm{a} / \mathrm{b}$ and of area 31 , respectively $(\boldsymbol{B}, \boldsymbol{C})$.

\section{Dorsal striatum}

The dorsal striatum mask, situated either side of the internal capsule, appeared to be predominantly interconnected with the dorsal supracallosal anterior cingulate cortex. Additional analysis confirmed a high probability of connection with the cingulate clusters on the anterior cingulate sulcus, clusters 3-6, but showed that there was a relatively high probability of connection in at least some voxels of several other clusters, especially subgenual cluster 1 (Fig. 5D, Table 1).

\section{Hypothalamus}

There was strong evidence of interconnection between subgenual and perigenual cingulate cortex and the hypothalamus. There was, however, also a weaker suggestion of interconnection with the ventral supracallosal region. Additional analysis suggested that clusters 1 and 2 had the highest probability of connection with the hypothalamus but that there was also some evidence of connection between the hypothalamus and ventral supracallosal clusters 7 and 8 (Fig. 5E, Table 1).

\section{Parietal cortex}

Voxels of high probability with the lateral parietal cortex were found spread across the midcingulate and posterior cingulate regions. Additional analysis suggested some overlap with the connections with the motor regions (precentral gyrus and premotor cortex): the parietal cortex also had a reasonably high probability of connection with cluster 6 in the posterior part of the anterior cingulate sulcus, and there was some evidence of connections with cluster 5 that lay immediately anteriorly. There was also evidence for connections with the clusters 8 and 9 and posterior parts of cluster 7 (Fig. 5F, Table 1).

\section{Orbitofrontal cortex}

Like the ventral striatum, medial orbitofrontal cortex connected with the highest probability to subgenual and pregenual anterior cingulate cortex. The highest connection probabilities were for clusters 1 and 2, with the highest relative contribution for cluster 2 (Fig. 5G, Table 1). The estimated pattern of connectivity between lateral orbitofrontal cortex and cingulate cortex was similar to that found between medial orbitofrontal cortex and cingulate cortex (Fig. $5 H)$. Despite the similarity in connection pattern, there was a noticeable difference in connection strength; the median number of connection streamlines estimated for each voxel in each cingulate cluster with the medial orbital region was $\sim 40$ times greater than the equivalent number for the lateral orbital region. In general, we refrain from comparing the connections of different extracingulate regions with one another because the comparison will be partly dependent on the size of the extracingulate target region and the route by which it interconnects with cingulate cortex. Nevertheless, it is of note that, despite the fact that these two extracingulate masks have very similar volumes, the medial orbitofrontal cortex was estimated to be much more strongly interconnected with the anterior cingulate cortex than was the adjacent lateral orbitofrontal cortex.

\section{Premotor cortex}

The premotor cortex had the highest probability of connection with dorsal supracallosal anterior cingulate cortex. Additional analysis demonstrated that the highest probability of interconnection was with the dorsal anterior cingulate clusters. The highest probability of connection was found for cluster 5 , followed by cluster 6 and then cluster 4 (Fig. 5I, Table 1).

\section{Precentral gyrus}

The precentral gyrus mask, which was located just posterior to the premotor mask, also had a high probability of interconnection with the dorsal anterior cingulate sulcus region. In the case of the precentral gyrus, however, the focus of connection probability was more posterior than had been the case for the premotor cortex. The highest probability of connection was with cluster 6 , followed by cluster 5 (Fig. 5J, Table 1). 


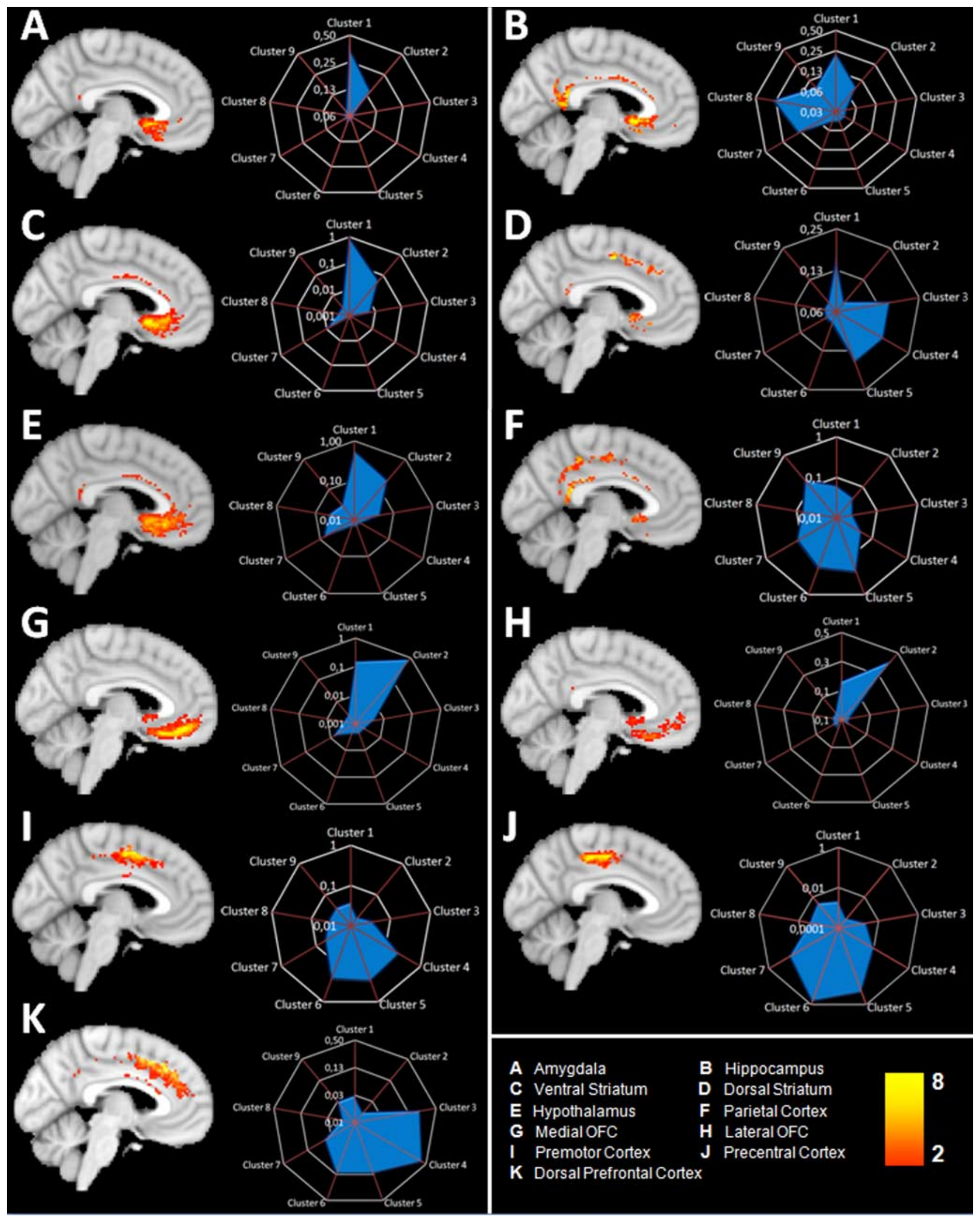

Figure 5. A, Group overlay of voxels with a high probability of interconnection with the amygdala, thresholded at no less than two subjects (thus excluding voxels that had survived initial thresholding in a single subject only). Note, however, that yellow voxels are ones in which connections to a given area were found in eight or more subjects. Amygdala connectivity fingerprint showing the relative connection probability between the amygdala and the nine cingulate/medial frontal clusters. The highest amygdala connection probabilities were with clusters 1, 2, and 7. Note that the connectivity fingerprint gives a threshold-free indication of the average strength (across all subjects) of connection between each cingulate/medial frontal cluster and each target region. Regions with a high probability of interconnection and connectivity fingerprints are shown for the hippocampus $(\boldsymbol{B})$, ventral striatum $(\boldsymbol{C})$, dorsal striatum $(\boldsymbol{D})$, hypothalamus $(\boldsymbol{E})$, parietal cortex $(\boldsymbol{F})$, medial orbitofrontal cortex $(\boldsymbol{G})$, lateral orbitofrontal cortex $(\boldsymbol{H})$, premotor cortex $(\boldsymbol{I})$, precentral cortex $(\boldsymbol{J})$, and dorsal prefrontal cortex $(\boldsymbol{K})$. Note that all fingerprint figures are plotted using a log scale, but that scaling varies. 
Table 1. Median normalized connection probabilities between each cingulate cluster and each extracingulate region

\begin{tabular}{|c|c|c|c|c|c|c|c|c|c|c|c|}
\hline Cluster & Amygdala & Hippocampus & Ventral striatum & Dorsal striatum & Hypothalamus & Parietal cortex & Medial OFC & Lateral OFC & Premotor cortex & Precentral cortex & Dorsal PFC \\
\hline 1 & 0.36 & 0.23 & 0.92 & 0.15 & 0.56 & 0.06 & 0.15 & 0.15 & 0.04 & 0.00 & 0.03 \\
\hline 2 & 0.14 & 0.09 & 0.05 & 0.08 & 0.20 & 0.04 & 0.83 & 0.38 & 0.02 & 0.00 & 0.01 \\
\hline 3 & 0.07 & 0.05 & 0.01 & 0.15 & 0.05 & 0.03 & 0.00 & 0.08 & 0.04 & 0.00 & 0.22 \\
\hline 4 & 0.07 & 0.05 & 0.00 & 0.15 & 0.02 & 0.05 & 0.00 & 0.08 & 0.25 & 0.01 & 0.40 \\
\hline 5 & 0.07 & 0.05 & 0.00 & 0.15 & 0.02 & 0.26 & 0.00 & 0.00 & 0.29 & 0.23 & 0.12 \\
\hline 6 & 0.07 & 0.05 & 0.00 & 0.08 & 0.02 & 0.21 & 0.00 & 0.08 & 0.25 & 0.68 & 0.12 \\
\hline 7 & 0.07 & 0.14 & 0.01 & 0.08 & 0.08 & 0.13 & 0.10 & 0.08 & 0.05 & 0.06 & 0.04 \\
\hline 8 & 0.07 & 0.27 & 0.00 & 0.08 & 0.05 & 0.07 & 0.00 & 0.08 & 0.04 & 0.00 & 0.01 \\
\hline 9 & 0.07 & 0.09 & 0.00 & 0.08 & 0.03 & 0.15 & 0.00 & 0.08 & 0.04 & 0.00 & 0.03 \\
\hline
\end{tabular}

The values represent the connection of each cluster probability as a proportion of total cingulate connections for a given extracingulate region. OFC, Orbitofrontal cortex; PFC, prefrontal cortex.

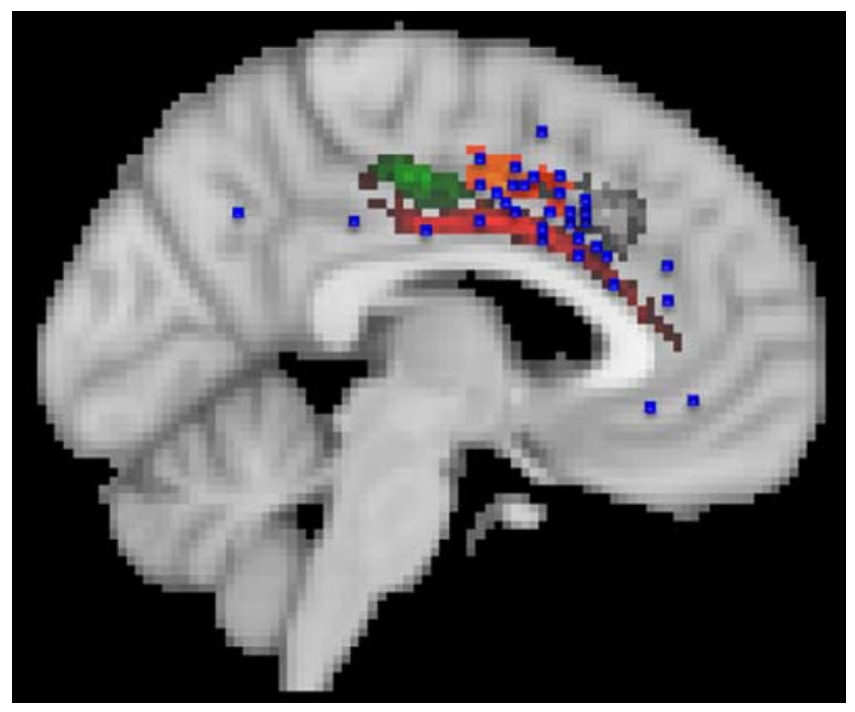

Figure 6. Peak activations from neuroimaging studies of pain are found predominantly in the supracallosal cingulate cortex (blue squares). Many of the activations are in the ventral part of this region in cluster 7, but some fall in the more anterior cingulate sulcus in cluster 5 . Cluster 5 may include the CMAr. Morrison and colleagues (Morrison and Downing, 2007; Morrison et al., 2007) have argued that rostral cingulate motor areas are also active when experimental participants are subjected to painful stimulation.

\section{Dorsal prefrontal cortex}

Evidence for dorsal prefrontal cortex connections was found throughout the cingulate cortex. The highest probability of connection, however, was with clusters 3 and 4 (Fig. $5 K$, Table 1 ).

\section{Meta-analysis of functional studies \\ Pain}

The results from 32 functional neuroimaging studies of pain were included in the meta-analysis. The vast majority of pain activations had coordinates in the mid supracallosal cingulate cortex (Fig. 6). Although many of the peaks were situated in the ventral part of the supracallosal region occupied by cluster 7 (33\% of activation peaks coincident with a cluster in more than six subjects were coincident with cluster 7), a number of the peaks were also found in the more dorsal anterior cingulate clusters, particularly cluster 5 (28\% of activation peaks coincident with a cluster in more than six subjects were coincident with cluster 5).

\section{Motor function}

The results from 23 neuroimaging studies of motor function were included in the meta-analysis. Activation related to motor function, as for pain, was again found predominantly in the mid supracallosal cingulate cortex (Fig. 7A). Motor-related activations, however, had a distinct pattern of localization (Figs. 6, 7A).
An ANOVA of the $x, y$, and $z$ coordinates associated with peaks of activation during pain and motor studies found a main effect of task condition $(F=4.642 ; \mathrm{df}=1,23 ; p=0.042)$ and an interaction between task condition and coordinate type $(F=4.270$; $\mathrm{df}=$ $2,46 ; p=0.038)$. A subsequent comparison of the $z$ coordinates associated with both types of task showed that the motor activation peaks were significantly more dorsal than the pain activation peaks $(t=-3.997$; $\mathrm{df}=54 ; p<0.001)$. They were most prominent in clusters 5,4 , and 6 [84\% of activation peaks coincident with a cluster in more than six subjects were coincident with either cluster $4(17 \%), 5(25 \%)$, or $6(46 \%)$ ].

There is evidence that two or three specialized subregions of the supracallosal cingulate sulcus in the monkey brain are interconnected with motor regions such as the ventral horn of the spinal cord, premotor cortex, and motor cortex (Mitz and Wise, 1987; Hutchins et al., 1988; Dum and Strick, 1991, 1993, 1996; Luppino et al., 1991; Shima et al., 1991; Morecraft and Van Hoesen, 1992; He et al., 1993; Van Hoesen et al., 1993; Galea and Darian-Smith, 1994; Morecraft et al., 2002). Previous metaanalyses of functional neuroimaging studies have therefore looked for evidence of cingulate regions with motor-related activity (Picard and Strick, 1996, 2001). One of the most influential, conducted by Picard and Strick (2001), argued for the existence of a "caudal cingulate [motor] zone" (CCZ) at the approximate anterior border of the most posterior of the cingulate sulcal clusters identified in the present study, cluster 6 . Cluster 6 was distinguished from the other motor-related regions in the supracallosal dorsal cingulate cortex by its high probability of interconnection with the motor cortex in precentral gyrus and with parietal cortex. In these two respects, it resembles the more caudal cingulate motor cortex of the macaque.

In addition, Picard and Strick found evidence for a "rostral cingulate [motor] zone." Picard and Strick attempted a finer coding of the motor tasks in their meta-analysis and argued that they could be subdivided into those in which "action selection" was manipulated and those in which activation related to "conflict" occurred. Although the number of experiments that were analyzed in this way was small, it was argued that the two foci suggest the further subdivision of the rostral cingulate motor zone (RCZ) into anterior and posterior parts, RCZa and RCZp. The present connectivity-based clustering approach offers some support for this argument because Picard and Strick's peak coordinates for RCZa and RCZp fall at the center of clusters 4 and 5, respectively (Fig. 7B).

\section{Conflict}

The results of 15 studies of conflict were included in the metaanalysis. The peaks of activation were situated in the anterior cingulate sulcus and paracingulate cortex (Fig. 7C). There was some overlap with the more anterior part of the distribution of the motor-related activations in cluster 4 (33\% of activation 
peaks coincident with a cluster in more than six subjects were coincident with cluster 4), but conflict-related activations were also found even more anteriorly in cluster 3 (33\% of activation peaks coincident with a cluster in more than six subjects were coincident with cluster 3). Like the meta-analysis of the previous study by Picard and Strick (2001), the results of the present, more extensive, meta-analysis suggest that the anterior cingulate sulcal tissue assigned to cluster 3 in the present study is affected by both motor task parameters and more "cognitive" manipulations. The median of coordinates, however, lay over the center of cluster 4 (Fig. $7 C$ ), as was the case in Picard and Strick's meta-analysis (Fig. 7B).

\section{Error detection}

The results of 16 studies of error detection were included in the meta-analysis. Activations were found in the dorsal anterior cingulate sulcus and paracingulate cortex. Error-related peak activations primarily overlapped with those related with conflict-related peak activations and were prominent in the vicinity of cluster 4 (Fig. 7D).

\section{Reward}

The results of 11 studies of reward were included in the meta-analysis. Some of the peak activations were found in the dorsal anterior cingulate sulcus and paracingulate cortex (Fig. 8), in which they overlapped with error-related activations in the vicinity of clusters 4 (17\% of activation peaks coincident with a cluster in more than six subjects were coincident with cluster 4$)$ and 3 (33\% of activation peaks coincident with a cluster in more than six subjects were coincident with cluster 3 ).

Additional analysis of activation patterns in the same dorsal anterior cingulate cortex region considered in light of both lesion and single-cell neurophysiology studies led to the proposal that activity here that precedes a decision encodes the integrated value of an action, whether in terms of immediate gains and costs or in terms of information to aid future decision making (Rushworth et al., 2007; Rushworth and Behrens, 2008). When the outcome of an action is detected, ACC activity encodes the prediction error, the difference between the reinforcement outcome that was expected and the reinforcement outcome that was received, and the degree to which the resulting information should influence future decisions (Behrens et al., 2007a). The same region is thus responsive to both error and reward feedback, as long as the feedback means that the expected value of an action is to be updated, both in humans and monkeys (Walton et al., 2004; Matsumoto et al., 2007; Sallet et al., 2007; Quilodran et al., 2008).

There was also, however, some evidence for a more ventromedial group of activations with a distinct focus in the pregenual and subgenual cortex of cluster 2 (50\% of activation peaks coincident with a cluster in more than six subjects were coincident with cluster 2). Unlike the more dorsal cingulate region, activity in this region is not closely tied to the selection of motor actions, and it is not clear that it encodes prediction errors but it has been shown to encode the expected value of a choice (Rushworth and Behrens, 2008).

Additional consideration of the papers in the meta-analysis revealed considerable variation in the naming of this brain region and that it has variously been referred to as anterior cingulate cortex, medial frontal cortex, ventromedial frontal cortex, and medial orbitofrontal cortex. It is therefore difficult to perform a meta-analysis of reward activations in this region using the same strict criteria as used elsewhere in the present report. Nevertheless, a survey of papers published in the same journals in the last 3 years that focused on reward expectation and often using computationally derived regressors to estimate subjects' reward expectations (Tanaka et al., 2004; Knutson and Cooper, 2005; Reuter et al., 2005; Daw and Doya, 2006; Hampton et al., 2006; Kim et al., 2006; Rolls et al., 2008) revealed the activations shown in yellow (Fig. 8) clustering around the same ventromedial region and cluster 2 . 


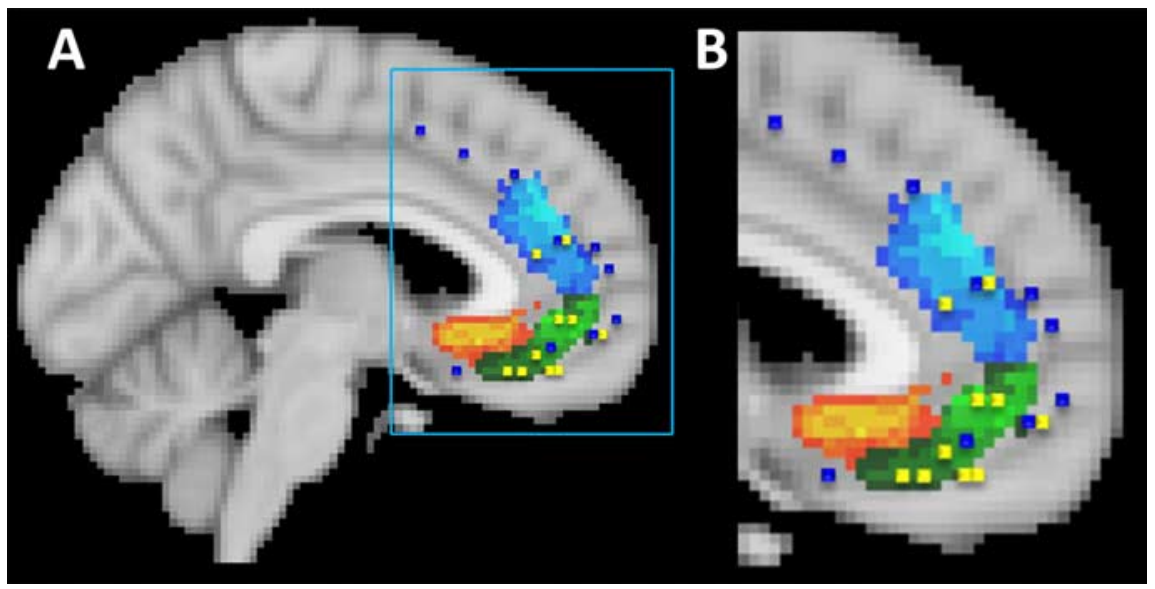

Figure 8. $\quad \boldsymbol{A}$, Reward-related activations were found in two regions in the meta-analysis (blue squares). First, there was a relatively dorsal region of activation in the dorsal anterior cingulate sulcus and paracingulate regions in clusters 4 and 3 . This is consistent with CMAr and the tissue immediately rostral to it being important for both reward and error processing in both macaques and humans (Walton et al., 2004; Kennerley et al., 2006). The second region was in the ventromedial frontal cortex and overlapped with cluster 2 . The picture of reward-related activation in this region provided by the meta-analysis may be incomplete because this region has been described variously as cingulate cortex, ventromedial frontal cortex, medial frontal cortex, and orbitofrontal cortex in different published reports. An additional survey of studies investigating reward and particularly reward expectation, often using computationally derived regressors that attempt to quantify the degree of reward expectation, found a number of additional activations in the cluster 2 region (shown in yellow). $\boldsymbol{B}$, Detail of $\boldsymbol{A}$.

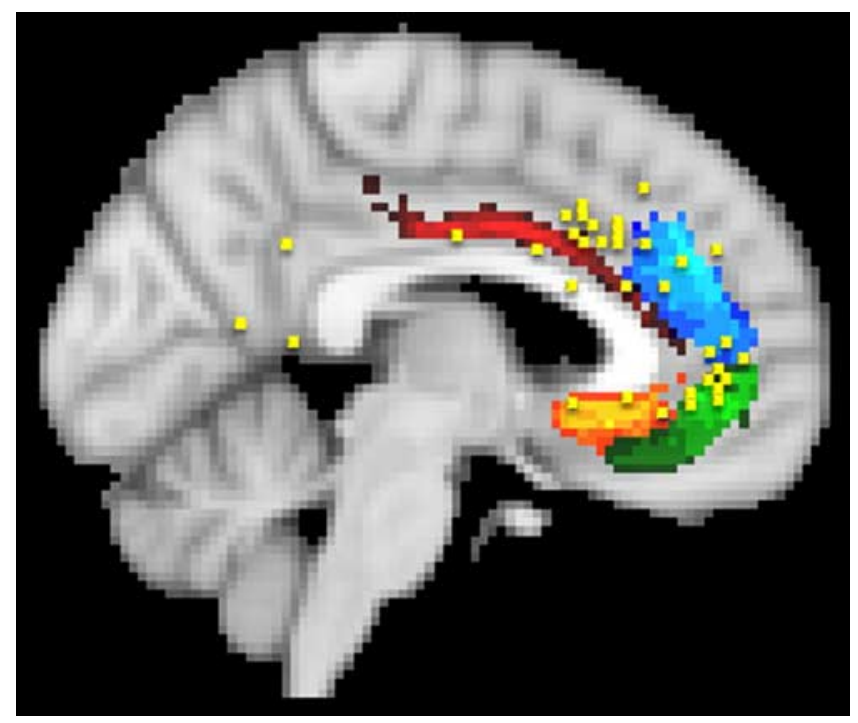

Figure 9. Emotion-related activations (yellow squares) overlapped with the positions of cluster 1 and the reward-related activations in cluster 2 .

\section{Emotion}

The results of 37 studies of emotion were included in the metaanalysis. Peak activation coordinates formed two distinct centers when projected onto the cingulate: one was found in the pregenual and subgenual anterior cingulate and one in the dorsal anterior cingulate cortex (Fig. 9). It has been argued that activation during social decision-making tasks that is seen in the first of these regions is related to the current reinforcement expectations, whereas activation in the more dorsal cingulate region is related to the saliency of the source of social information (Behrens et al., 2008). Although the dorsal anterior cingulate cortex region of emotion-related activation was adjacent to the motor-related region (compare Figs. 7, 9) it was still clearly distinct from it (interaction of task condition and coordinate position, $F=106.529$; $\mathrm{df}=2,26 ; p<0.001)$. Even in the dorsal cingulate cortex, a comparison of $y$ - and $z$-coordinate positions revealed that emotion-related activations occupied a significantly more anterior and ventral position $(t=8.181, \mathrm{df}=$ $18, p<0.001$; and $t=17.186, \mathrm{df}=36, p<$ 0.001 , respectively). Such a separation is consistent with the motor-related activations lying in the anterior cingulate sulcus in clusters 4-6, whereas the emotionrelated activations lie in cluster 7 on the cingulate gyrus (although only 17\% of activation peaks coincident with a cluster in more than six subjects were coincident with cluster 7 ). The location of even the more dorsal cluster of emotion-related activations was also clearly separable from the pain-related activations $(F=51.779$; $\mathrm{df}=2,26 ; p<0.001)$. A comparison of $y$-coordinate positions showed that the emotion-related activations were significantly more anteriorly positioned $(t=$ 5.882; $\mathrm{df}=44 ; p<0.001)$. The pregenual emotion activation region overlapped with the most dorsal part of cluster 2 and with cluster 3 ( $29 \%$ of activation peaks coincident with a cluster in more than six subjects were coincident with cluster 2 and $25 \%$ were coincident with cluster 3) (Fig. 9). Although there was a tendency for the emotion-related activations to lie dorsal to the reward-related activations, it did not quite reach statistical significance $(p>$ $0.05)$.

\section{Memory}

Results of 27 functional studies were included in the metaanalysis. For this condition, two spatially distinct centers of activation were found, one in the dorsal anterior cingulate cortex (19\% of activation peaks coincident with a cluster in more than six subjects were coincident with cluster 3 and 25\% were coincident with cluster 4 ) and one in the retrosplenial posterior cingulate cortex (19\% of activation peaks coincident with a cluster in more than six subjects were coincident with cluster 9). There is overlap between the anterior activations and activations seen in studies of error, reward, and conflict monitoring. Although a firm conclusion cannot be drawn from the current meta-analysis, it seems possible that the anterior region of activation reflects processes concerned with monitoring the success of memory retrieval or encoding. The posterior region of activation was quite distinct. It was similar to the region that, in the right hemisphere, has been implicated in spatial navigation possibly as a consequence of a role it may have as a store of spatial information or because it is involved in memory retrieval (Maguire, 2001; Spiers and Maguire, 2007). It has been argued that the corresponding region in the left hemisphere is concerned with more general aspects of episodic memory (Maguire, 2001). This area of activation overlapped with clusters 8 and 9 (Fig. 10A) and the posterior cingulate region associated with a high probability of interconnection with the hippocampus (Fig. $10 \mathrm{~B}$ ).

\section{Discussion}

Previous DWI-tractography parcellation studies have divided regions into two or three components, but, in the present study, a large region was divided into nine clusters in 11 subjects. Subgenual cluster 1 resembled one identified by Johansen-Berg et al. 
(2008) in a study that focused on the cortex targeted in deep brain stimulation treatments for depression (Mayberg et al., 2005). The position of cluster 1 and connections with hypothalamus, amygdala, hippocampus, ventral striatum, and orbitofrontal cortex make it similar to area 25 (Petrides and Pandya, 1994; Vogt et al., 1995). Changes in activity and structure, especially in amygdala and perigenual cingulate cortex, have been reported in depression (Mayberg et al., 2005; Pezawas et al., 2005). Price (2005) argued that its anatomical connections mean that it has a role in monitoring and regulating emotional and visceral states.

Cluster 7 extended across the anterior cingulate gyrus dorsal to the corpus callo-

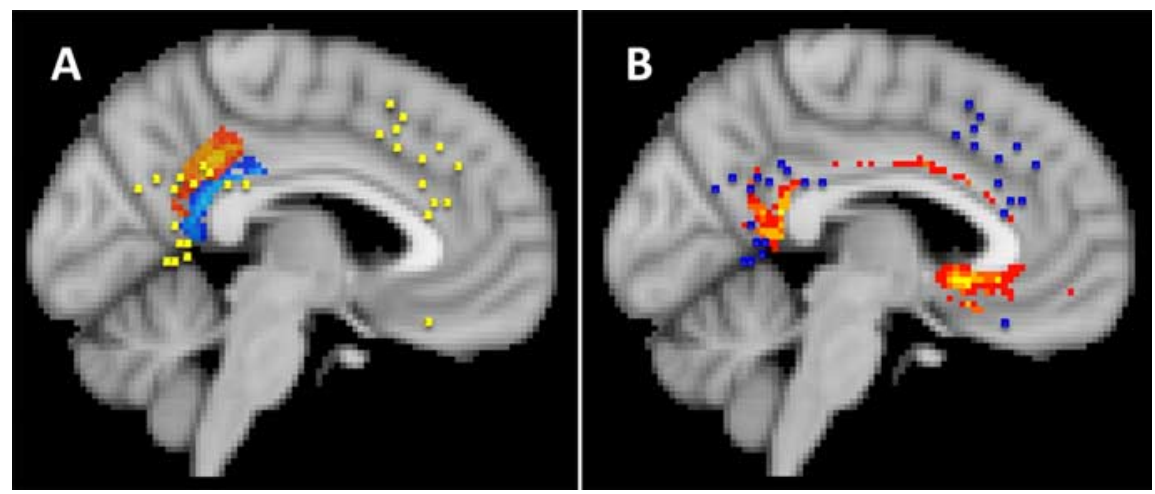

Figure 10. $\quad \boldsymbol{A}$, Two regions of memory-related activations (yellow squares in $\boldsymbol{A}$, blue squares in $\boldsymbol{B}$ ) were found. The more anterior region overlapped with error- and conflict-related activations but a distinct posterior region overlapping with retrosplenial clusters 8 and 9 was also activated by memory tasks. $\boldsymbol{B}$, The same posterior cingulate region that was associated with memory was also associated with a high probability of connection to the hippocampus. sum and into pregenual cortex. Its position resembles that of area 24 (Petrides and Pandya, 1994; Vogt et al., 1995; Ongür et al., 2003). Area 24 can be further subdivided into anterior and posterior subregions in humans (Vogt et al., 1995) and monkeys (Vogt et al., 2005), and future studies may reveal differences in DWI-derived probabilistic connectivity within cluster 7 . The observation that fMRI-measured activity levels at several points in cluster 7 are positively correlated with one another (Margulies et al., 2007) is, nevertheless, consistent with cortex throughout this region sharing many anatomical inputs. The functional meta-analysis demonstrated that cluster 7 was associated with pain and emotion, but there was significant separation between these activations (Vogt et al., 2005). In the macaque, lesions that include gyral area 24 cause less value to be assigned to social information (Rudebeck et al., 2006). In agreement, human neuroimaging studies emphasize cluster 7 and adjacent cluster 3 in social interaction (King-Casas et al., 2005; Tomlin et al., 2006). Again, in the human, the region has a key role in the assignment of value to social information (Behrens et al., 2008). The connections of this area, with hypothalamus, amygdala, ventral striatum, and orbitofrontal cortex, are consistent with those reported in macaque (Porrino et al., 1981; Vogt and Pandya, 1987; Vogt et al., 1987; Van Hoesen et al., 1993; Ongür et al., 1998) and a role in emotion and social behavior.

More dorsal supracallosal cingulate cortex is involved in very distinct functions (Bush et al., 2000). The functional metaanalysis implicated all of this region in motor control and suggested the most anterior part monitors action errors and conflict (Botvinick, 2007) and is important for reinforcement-guided action selection (Rushworth et al., 2007). Consistent with its distinct function, the dorsal region was composed of different clusters, clusters 4-6, with distinct connections, with lateral premotor cortex, that are not shared by ventral cingulate cortex. Such a profile suggests a correspondence to the CMAs in macaque (Paus, 2001; Picard and Strick, 2001) in specialized subdivisions of areas 24 and possibly areas 23, 24c, 24c' , and 23c (Vogt et al., 1995).

Picard and Strick (2001) argued that two regions, RCZa and RCZp, existed in anterior dorsal cingulate. Their median RCZa and RCZp coordinates fall at the centers of clusters 4 and 5, respectively. As in the human (Walton et al., 2004), the corresponding region in macaque is involved in reinforcement-guided action selection (Kennerley et al., 2006; Quilodran et al., 2008). The high probability of connection between cluster 4 and dorsal prefrontal cortex also makes it similar to CMAr. The more pos- terior cluster 6 resembles a third region proposed by Picard and Strick, the CCZ. Its higher probability of connection with parietal and motor cortex is consistent with the CCZ identification and a role in controlling basic aspects of movement.

The location of cluster 3 resembles that of area 32 (Brodmann, 1909; Petrides and Pandya, 1994). Some refer to this region as $32 \mathrm{ac}, 32 \mathrm{~h}$, or $32 \mathrm{p}$ to indicate its separation from subgenual area 32 (Ongür et al., 2003; Palomero-Gallagher et al., 2008). It had a high probability of interconnection with ventral and dorsal striatum and orbitofrontal cortex. Cluster 3 is the most difficult cluster to relate to macaque. Some error monitoring activations were found here, suggesting that it may be an expansion of tissue immediately anterior to CMAr. Several studies have reported activation in this region and its anterior border during social interaction and theory of mind tasks (Amodio and Frith, 2006; Gilbert et al., 2006). Macaque anterior cingulate cortex is also activated by social stimuli (Gil-da-Costa et al., 2004).

Posterior cingulate cortex is fundamentally distinct in function to anterior cingulate cortex, and roles in spatial orientation and memory have been emphasized (Vogt et al., 1992; Spiers and Maguire, 2007). The current meta-analysis confirmed its activation during memory tasks. It contained two clusters, 8 and 9 , with positions resembling those of cytoarchitectonic areas $23 \mathrm{a} / 23 \mathrm{~b}$ and 31, respectively (Vogt et al., 1995, 2005). Cytoarchitectonic studies have identified two small areas, 29 and 30, in the callosal sulcus. Although these areas were difficult to identify in the present study, they are known to be interconnected with the subiculum (Rosene and Van Hoesen, 1977). Consistent with anatomical observations in macaque and the activation of this region in memory tasks, there was evidence of hippocampal connectivity with ventral posterior cingulate cortex in the present study. Again, as in macaque (Parvizi et al., 2006) and consistent with a spatial function, posterior cingulate cortex had a high probability of interconnection with lateral parietal cortex.

A ventromedial frontal cluster, cluster 2, was also identified. Several anatomists report that tissue in this region is dysgranular and label it area 32 (Petrides and Pandya, 1994; Vogt et al., 1995). In recognition of differences between its cytoarchitecture and that of more dorsal 32, it has been labeled area 32s (PalomeroGallagher et al., 2008). Ongür et al. (2003) also note the distinct nature of tissue in this region but label it medial frontopolar area 10, 10m (Ongür et al., 2003). Area 10 extends posteriorly between cingulate and orbitofrontal cortex in macaque (Carmichael and Price, 1994; Vogt et al., 2005), and, as in macaque (Carmichael 
and Price, 1995; Petrides and Pandya, 2007), there was evidence of cluster 2 interconnection with amygdala, orbitofrontal cortex, and hypothalamus. The area was associated with reward and reward expectation in the functional meta-analysis. Although activations in this region are often related to orbitofrontal cortex, the present results are consistent with it being a distinct area.

A number of findings now attest to the reliability of DWI tractography. DWI-derived boundaries are replicable across scanning sessions both within and across individuals (Klein et al., 2007; Tomassini et al., 2007). Parcellation clusters are correlated with regional differences in functional activation in the same subjects (Johansen-Berg et al., 2004). Moreover, statistical differences in connectivity profiles in meta-analyses of animal tract tracing studies are related to differences in function (Stephan et al., 2000; Kötter et al., 2001; Passingham et al., 2002; Averbeck and Seo, 2008).

The DWI-tractography procedure is, however, probabilistic and insensitive to several features of axonal projections, such as polarity, that can be examined with tract tracing techniques in animals, which remain the gold standard for studying anatomical connectivity in the primate brain. Nevertheless, in macaques, DWI tractography has identified several frontal cortical connections known from tract tracing studies (Croxson et al., 2005), and individual differences in DWI-estimated connection strength are correlated with neurophysiological indices of connectivity (Boorman et al., 2007; Wahl et al., 2007).

Although tract tracing techniques yield unambiguous information about connections, the probability values derived from DWI tractography are influenced not only by the true underlying probability of an anatomical connection existing but also by other factors, such as the size of the target mask, its distance from the seed mask, and the geometry of the pathways between the seed and target (more tortuous, or crossing, pathways will be harder to track). This is why we have been wary of reading too much into quantitative differences in absolute connectivity values but rather have focused on differences in patterns of relative connection strength.

DWI tractography provides less information than cytoarchitecture. For example, during parcellation, small subcallosal sulcal areas were not identified. This may, however, just reflect these small size of the areas, their proximity to the corpus callosum, and the care taken to avoid including callosal tissue in the cingulate mask. In addition, although an area with a high amygdala connection probability was identified that resembled the core amygdala-connecting region in macaque, there was no strong evidence for interconnection between dorsal cingulate motor regions and amygdala in the present study, although such connections exist, albeit at a lower density, in the macaque (Morecraft et al., 2007). It should, however, be noted that the region identified as interconnected with amygdala in the present study resembles that found to vary in tandem with amygdala in morphometric analyses of the human brain (Pezawas et al., 2005). Despite these limitations, DWI tractography has the advantage that it can be used in vivo, and so it was possible to demonstrate that principal features of cingulate anatomy established in other primates are also present in the human.

\section{References}

Alkadhi H, Crelier GR, Boendermaker SH, Hepp-Reymond MC, Kollias SS (2002a) Somatotopy in the ipsilateral primary motor cortex. Neuroreport 13:2065-2070.

Alkadhi H, Crelier GR, Boendermaker SH, Golay X, Hepp-Reymond MC, Kollias SS (2002b) Reproducibility of primary motor cortex somatotopy under controlled conditions. Am J Neuroradiol 23:1524-1532.
Amaral DG, Price JL (1984) Amygdalo-cortical projections in the monkey (Macaca fascicularis). J Comp Neurol 230:465-496.

Amodio DM, Frith CD (2006) Meeting of minds: the medial frontal cortex and social cognition. Nat Rev Neurosci 7:268-277.

Anwander A, Tittgemeyer M, von Cramon DY, Friederici AD, Knösche TR (2007) Connectivity-based parcellation of Broca's area. Cereb Cortex $17: 816-825$.

Averbeck BB, Seo M (2008) The statistical neuroanatomy of frontal networks in the macaque. PLoS Comput Biol 4:e1000050.

Baleydier C, Mauguiere F (1980) The duality of the cingulate gyrus in monkey. Neuroanatomical study and functional hypothesis. Brain 103:525-554.

Barbas H, Pandya DN (1987) Architecture and frontal cortical connections of the premotor cortex (area 6) in the rhesus monkey. J Comp Neurol 256:211-228.

Barbas H, Ghashghaei H, Dombrowski SM, Rempel-Clower NL (1999) Medial prefrontal cortices are unified by common connections with superior temporal cortices and distinguished by input from memory related areas in the rhesus monkey. J Comp Neurol 410:343-367.

Bates JF, Goldman-Rakic PS (1993) Prefrontal connections of medial motor areas in the rhesus monkey. J Comp Neurol 336:211-228.

Behrens TE, Johansen-Berg H, Woolrich MW, Smith SM, Wheeler-Kingshott CA, Boulby PA, Barker GJ, Sillery EL, Sheehan K, Ciccarelli O, Thompson AJ, Brady JM, Matthews PM (2003) Non-invasive mapping of connections between human thalamus and cortex using diffusion imaging. Nat Neurosci 6:750-757.

Behrens TE, Woolrich MW, Walton ME, Rushworth MF (2007a) Learning the value of information in an uncertain world. Nat Neurosci 10:1214-1221.

Behrens TE, Berg HJ, Jbabdi S, Rushworth MF, Woolrich MW (2007b) Probabilistic diffusion tractography with multiple fibre orientations: what can we gain? Neuroimage 34:144-155.

Behrens TE, Hunt LT, Woolrich MW, Rushworth MF (2008) Associative learning of social value. Nature 456:245-249.

Boorman ED, O'Shea J, Sebastian C, Rushworth MF, Johansen-Berg H (2007) Individual differences in white-matter microstructure reflect variation in functional connectivity during choice. Curr Biol 17:1426-1431.

Botvinick MM (2007) Conflict monitoring and decision making: reconciling two perspectives on anterior cingulate function. Cogn Affect Behav Neurosci 7:356-366.

Botvinick MM, Cohen JD, Carter CS (2004) Conflict monitoring and anterior cingulate cortex: an update. Trends Cogn Sci 8:539-546.

Brodmann K (1909) Vergleichende Lokalisationslehre der Grosshirnrinde in ihren Prinzipien dargestellt auf Grund des Zellenbaues. Liepzig, Germany: J. A. Barth [translated as Localisation in the Cerebral Cortex, by L. J. Garey (1994). London: Smith-Gordon].

Bush G, Luu P, Posner MI (2000) Cognitive and emotional influences in anterior cingulate cortex. Trends Cogn Sci 4:215-222.

Carmichael ST, Price JL (1994) Architectonic subdivision of the orbital and medial prefrontal cortex in the macaque monkey. J Comp Neurol 346:366-402.

Carmichael ST, Price JL (1995) Limbic connections of the orbital and medial prefrontal cortex in macaque monkeys. J Comp Neurol 363:615-641.

Carmichael ST, Price JL (1996) Connectional networks within the orbital and medial prefrontal cortex of macaque monkeys. J Comp Neurol 371:179-207.

Cavada C, Goldman-Rakic PS (1989) Posterior parietal cortex in rhesus monkey. I. Parcellation of areas based on distinctive limbic and sensory corticocortical connections. J Comp Neurol 287:393-421.

Cavada C, Compañy T, Tejedor J, Cruz-Rizzolo RJ, Reinoso-Suárez F (2000) The anatomical connections of the macaque monkey orbitofrontal cortex. A review. Cereb Cortex 10:220-242.

Chiavaras MM, Petrides M (2000) Orbitofrontal sulci of the human and macaque monkey brain. J Comp Neurol 422:35-54.

Chiavaras MM, LeGoualher G, Evans A, Petrides M (2001) Threedimensional probabilistic atlas of the human orbitofrontal sulci in standardized stereotaxic space. Neuroimage 13:479-496.

Collins DL, Neelin P, Peters TM, Evans AC (1994) Automatic 3D intersubject registration of MR volumetric data in standardized Talairach space. J Comput Assist Tomogr 18:192-205.

Critchley HD, Corfield DR, Chandler MP, Mathias CJ, Dolan RJ (2000) 
Cerebral correlates of autonomic cardiovascular arousal: a functional neuroimaging investigation in humans. J Physiol 523:259-270.

Croxson PL, Johansen-Berg H, Behrens TE, Robson MD, Pinsk MA, Gross CG, Richter W, Richter MC, Kastner S, Rushworth MF (2005) Quantitative investigation of connections of the prefrontal cortex in the human and macaque using probabilistic diffusion tractography. J Neurosci 25:8854-8866.

Daw ND, Doya K (2006) The computational neurobiology of learning and reward. Curr Opin Neurobiol 16:199-204.

Dum RP, Strick PL (1991) The origin of corticospinal projections from the premotor areas in the frontal lobe. J Neurosci 11:667-689.

Dum RP, Strick PL (1993) Cingulate motor areas. In: Cingulate cortex and limbic thalamus (Vogt BA, Gabriel M, eds), pp 415-441. Boston: Birkhäuser.

Dum RP, Strick PL (1996) Spinal cord terminations of the medial wall motor areas in macaque monkeys. J Neurosci 16:6513-6525.

Freedman LJ, Insel TR, Smith Y (2000) Subcortical projections of area 25 (subgenual cortex) of the macaque monkey. J Comp Neurol 421:172-188.

Galea MP, Darian-Smith I (1994) Multiple corticospinal neuron populations in the macaque monkey are specified by their unique cortical origins, spinal terminations, and connections. Cereb Cortex 4:166-194.

Geyer S, Matelli M, Luppino G, Zilles K (2000) Functional neuroanatomy of the primate isocortical motor system. Anat Embryol (Berl) 202:443-474.

Ghashghaei HT, Hilgetag CC, Barbas H (2007) Sequence of information processing for emotions based on the anatomic dialogue between prefrontal cortex and amygdala. Neuroimage 34:905-923.

Gilbert SJ, Spengler S, Simons JS, Steele JD, Lawrie SM, Frith CD, Burgess PW (2006) Functional specialization within rostral prefrontal cortex (area 10): a meta-analysis. J Cogn Neurosci 18:932-948.

Gil-da-Costa R, Braun A, Lopes M, Hauser MD, Carson RE, Herscovitch P, Martin A (2004) Toward an evolutionary perspective on conceptual representation: species-specific calls activate visual and affective processing systems in the macaque. Proc Natl Acad Sci U S A 101:17516-17521.

Gotlib IH, Sivers H, Gabrieli JD, Whitfield-Gabrieli S, Goldin P, Minor KL, Canli T (2005) Subgenual anterior cingulate activation to valenced emotional stimuli in major depression. Neuroreport 16:1731-1734.

Haber SN, Kunishio K, Mizobuchi M, Lynd-Balta E (1995) The orbital and medial prefrontal circuit through the primate basal ganglia. J Neurosci 15:4851-4867.

Haber SN, Kim KS, Mailly P, Calzavara R (2006) Reward-related cortical inputs define a large striatal region in primates that interface with associative cortical connections, providing a substrate for incentive-based learning. J Neurosci 26:8368-8376.

Hampton AN, Bossaerts P, O'Doherty JP (2006) The role of the ventromedial prefrontal cortex in abstract state-based inference during decision making in humans. J Neurosci 26:8360-8367.

Hatanaka N, Tokuno H, Hamada I, Inase M, Ito Y, Imanishi M, Hasegawa N, Akazawa T, Nambu A, Takada M (2003) Thalamocortical and intracortical connections of monkey cingulate motor areas. J Comp Neurol 462:121-138.

He SQ, Dum RP, Strick PL (1993) Topographic organization of corticospinal projections from the frontal lobe: motor areas on the lateral surface of the hemisphere. J Neurosci 13:952-980.

He SQ, Dum RP, Strick PL (1995) Topographic organization of corticospinal projections from the frontal lobe: motor areas on the medial surface of the hemisphere. J Neurosci 15:3284-3306.

Holroyd CB, Coles MG (2002) The neural basis of human error processing: reinforcement learning, dopamine, and the error-related negativity. Psychol Rev 109:679-709.

Husain M, Nachev P (2007) Space and the parietal cortex. Trends Cogn Sci 11:30-36.

Hutchins KD, Martino AM, Strick PL (1988) Corticospinal projections from the medial wall of the hemisphere. Exp Brain Res 71:667-672.

Johansen-Berg H, Behrens TE, Robson MD, Drobnjak I, Rushworth MF, Brady JM, Smith SM, Higham DJ, Matthews PM (2004) Changes in connectivity profiles define functionally distinct regions in human medial frontal cortex. Proc Natl Acad Sci U S A 101:13335-13340.

Johansen-Berg H, Gutman DA, Behrens TE, Matthews PM, Rushworth MF, Katz E, Lozano AM, Mayberg HS (2008) Anatomical connectivity of the subgenual cingulate region targeted with deep brain stimulation for treatment-resistant depression. Cereb Cortex 18:1374-1383.
Jones EG, Powell TPS (1970) An anatomical study of converging sensory pathways within the cerebral cortex of the monkey. Brain 93:793-820.

Kennerley SW, Walton ME, Behrens TE, Buckley MJ, Rushworth MF (2006) Optimal decision making and the anterior cingulate cortex. Nat Neurosci 9:940-947.

Kim H, Shimojo S, O’Doherty JP (2006) Is avoiding an aversive outcome rewarding? Neural substrates of avoidance learning in the human brain. PLoS Biol 4:e233.

King-Casas B, Tomlin D, Anen C, Camerer CF, Quartz SR, Montague PR (2005) Getting to know you: reputation and trust in a two-person economic exchange. Science 308:78-83.

Klein JC, Behrens TE, Robson MD, Mackay CE, Higham DJ, Johansen-Berg H (2007) Connectivity-based parcellation of human cortex using diffusion MRI: Establishing reproducibility, validity and observer independence in BA 44/45 and SMA/pre-SMA. Neuroimage 34:204-211.

Knutson B, Cooper JC (2005) Functional magnetic resonance imaging of reward prediction. Curr Opin Neurol 18:411-417.

Kötter R, Stephan KE, Palomero-Gallagher N, Geyer S, Schleicher A, Zilles K (2001) Multimodal characterisation of cortical areas by multivariate analyses of receptor binding and connectivity data. Anat Embryol (Berl) 204:333-350.

Kubicki M, McCarley R, Westin CF, Park HJ, Maier S, Kikinis R, Jolesz FA, Shenton ME (2005) A review of diffusion tensor imaging studies in schizophrenia. J Psychiatr Res 41:15-30.

Kunishio K, Haber SN (1994) Primate cingulostriatal projection: limbic striatal versus sensorimotor striatal input. J Comp Neurol 350:337-356.

Lu MT, Preston JB, Strick PL (1994) Interconnections between the prefrontal cortex and the premotor areas in the frontal lobe. J Comp Neurol 341:375-392.

Luppino G, Matelli M, Camarda RM, Gallese V, Rizzolatti G (1991) Multiple representations of body movements in mesial area 6 and the adjacent cingulate cortex: an intracortical microstimulation study in the macaque monkey. J Comp Neurol 311:463-482.

Luppino G, Matelli M, Camarda R, Rizzolatti G (1994) Corticospinal projections from mesial frontal and cingulate areas in the monkey. Neuroreport 5:2545-2548.

Luppino G, Govoni P, Matelli M (1998) Prefrontal and cingulate afferents to the rostral premotor areas in the macaque monkey. Soc Neurosci Abstr 24:257.211.

Luppino G, Rozzi S, Calzavara R, Matelli M (2003) Prefrontal and agranular cingulate projections to the dorsal premotor areas F2 and F7 in the macaque monkey. Eur J Neurosci 17:559-578.

Maguire EA (2001) The retrosplenial contribution to human navigation: a review of lesion and neuroimaging findings. Scand J Psychol 42:225-238.

Margulies DS, Kelly AM, Uddin LQ, Biswal BB, Castellanos FX, Milham MP (2007) Mapping the functional connectivity of anterior cingulate cortex. Neuroimage 37:579-588.

Matelli M, Luppino G, Rizzolatti G (1991) Architecture of superior and mesial area 6 and the adjacent cingulate cortex in the macaque monkey. J Comp Neurol 311:445-462.

Matsumoto M, Matsumoto K, Abe H, Tanaka K (2007) Medial prefrontal cell activity signaling prediction errors of action values. Nat Neurosci 10:647-656.

Mayberg HS, Lozano AM, Voon V, McNeely HE, Seminowicz D, Hamani C, Schwalb JM, Kennedy SH (2005) Deep brain stimulation for treatmentresistant depression. Neuron 45:651-660.

Mitz AR, Wise SP (1987) The somatotopic organization of the supplementary motor area: intracortical microstimulation mapping. J Neurosci 7:1010-1021.

Morecraft RJ, Van Hoesen GW (1992) Cingulate input to the primary and supplementary motor cortices in the rhesus monkey: evidence for somatotopy in areas 24c and 23c. J Comp Neurol 322:471-489.

Morecraft RJ, Van Hoesen GW (1993) Frontal granular cortex input to the cingulate (M3), supplementary (M2) and primary (M1) motor cortices in the rhesus monkey. J Comp Neurol 337:669-689.

Morecraft RJ, Schroeder CM, Keifer J (1996) Organization of face representation in the cingulate cortex of the rhesus monkey. Neuroreport 7:1343-1348.

Morecraft RJ, Herrick JL, Stilwell-Morecraft KS, Louie JL, Schroeder CM, Ottenbacher JG, Schoolfield MW (2002) Localization of arm representation in the corona radiata and internal capsule in the non-human primate. Brain 125:176-198. 
Morecraft RJ, Cipolloni PB, Stilwell-Morecraft KS, Gedney MT, Pandya DN (2004) Cytoarchitecture and cortical connections of the posterior cingulate and adjacent somatosensory fields in the rhesus monkey. J Comp Neurol 469:37-69.

Morecraft RJ, McNeal DW, Stilwell-Morecraft KS, Gedney M, Ge J, Schroeder CM, van Hoesen GW (2007) Amygdala interconnections with the cingulate motor cortex in the rhesus monkey. J Comp Neurol 500:134-165.

Morrison I, Downing PE (2007) Organization of felt and seen pain responses in anterior cingulate cortex. Neuroimage 37:642-651.

Morrison I, Peelen MV, Downing PE (2007) The sight of others' pain modulates motor processing in human cingulate cortex. Cereb Cortex 17:2214-2222.

Naggara O, Oppenheim C, Rieu D, Raoux N, Rodrigo S, Dalla Barba G, Meder JF (2006) Diffusion tensor imaging in early Alzheimer's disease. Psychiatry Res 146:243-249.

Ongür D, Price JL (2000) The organization of networks within the orbital and medial prefrontal cortex of rats, monkeys and humans. Cereb Cortex 10:206-219.

Ongür D, An X, Price JL (1998) Orbital and medial prefrontal cortical projections to the hypothalamus in macaque monkeys. J Comp Neurol 401:480-505.

Ongür D, Ferry AT, Price JL (2003) Architectonic subdivision of the human orbital and medial prefrontal cortex. J Comp Neurol 460:425-449.

Palomero-Gallagher N, Mohlberg H, Zilles K, Vogt B (2008) Cytology and receptor architecture of human anterior cingulate cortex. J Comp Neurol 508:906-926.

Parvizi J, Van Hoesen GW, Buckwalter J, Damasio A (2006) Neural connections of the posteromedial cortex in the macaque. Proc Natl Acad Sci U S A 103:1563-1568.

Passingham RE, Stephan KE, Kötter R (2002) The anatomical basis of functional localization in the cortex. Nat Rev Neurosci 3:606-616.

Paus T (2001) Primate anterior cingulate cortex: where motor control, drive and cognition interface. Nat Rev Neurosci 2:417-424.

Paus T, Tomaiuolo F, Otaky N, MacDonald D, Petrides M, Atlas J, Morris R, Evans AC (1996a) Human cingulate and paracingulate sulci: pattern, variability, asymmetry, and probabilistic map. Cereb Cortex 6:207-214.

Paus T, Otaky N, Caramanos Z, MacDonald D, Zijdenbos A, D’Avirro D, Gutmans D, Holmes C, Tomaiuolo F, Evans AC (1996b) In vivo morphometry of the intrasulcal gray matter in the human cingulate, paracingulate, and superior-rostral sulci: hemispheric asymmetries, gender differences and probability maps. J Comp Neurol 376:664-673.

Petrides M, Pandya DN (1994) Comparative architectonic analysis of the human and the macaque frontal cortex. In: Handbook of neuropsychology (Boller F, Grafman J, eds), pp 17-58. Amsterdam: Elsevier Science.

Petrides M, Pandya DN (2007) Efferent association pathways from the rostral prefrontal cortex in the macaque monkey. J Neurosci 27:11573-11586.

Pezawas L, Meyer-Lindenberg A, Drabant EM, Verchinski BA, Munoz KE, Kolachana BS, Egan MF, Mattay VS, Hariri AR, Weinberger DR (2005) 5-HTTLPR polymorphism impacts human cingulate-amygdala interactions: a genetic susceptibility mechanism for depression. Nat Neurosci $8: 828-834$.

Picard N, Strick PL (1996) Motor areas of the medial wall: a review of their location and functional activation. Cereb Cortex 6:342-353.

Picard N, Strick PL (2001) Imaging the premotor areas. Curr Opin Neurobiol 11:663-672.

Porrino LJ, Crane AM, Goldman-Rakic PS (1981) Direct and indirect pathways from the amygdala to the frontal lobe in rhesus monkeys. J Comp Neurol 198:121-136.

Price JL (2005) Free will versus survival: brain systems that underlie intrinsic constraints on behavior. J Comp Neurol 493:132-139.

Pruessner JC, Köhler S, Crane J, Pruessner M, Lord C, Byrne A, Kabani N, Collins DL, Evans AC (2002) Volumetry of temporopolar, perirhinal, entorhinal and parahippocampal cortex from high-resolution MR images: considering the variability of the collateral sulcus. Cereb Cortex 12:1342-1353.

Quilodran R, Rothé M, Procyk E (2008) Behavioral shifts and action valuation in the anterior cingulate cortex. Neuron 57:314-325.

Reuter J, Raedler T, Rose M, Hand I, Gläscher J, Büchel C (2005) Pathological gambling is linked to reduced activation of the mesolimbic reward system. Nat Neurosci 8:147-148.

Rolls ET, McCabe C, Redoute J (2008) Expected value, reward outcome, and temporal difference error representations in a probabilistic decision task. Cereb Cortex 18:652-663.

Rosenberg DR, Mirza Y, Russell A, Tang J, Smith JM, Banerjee SP, Bhandari R, Rose M, Ivey J, Boyd C, Moore GJ (2004) Reduced anterior cingulate glutamatergic concentrations in childhood OCD and major depression versus healthy controls. J Am Acad Child Adolesc Psychiatry 43:1146-1153.

Rosene DL, Van Hoesen GW (1977) Hippocampal efferents reach widespread areas of cerebral cortex and amygdala in the rhesus monkey. Science 198:315-317.

Rudebeck PH, Buckley MJ, Walton ME, Rushworth MF (2006) A role for the macaque anterior cingulate gyrus in social valuation. Science 313:1310-1312.

Rushworth MF, Behrens TE (2008) Choice, uncertainty and value in prefrontal and cingulate cortex. Nat Neurosci 11:389-397.

Rushworth MF, Behrens TE, Johansen-Berg H (2006) Connection patterns distinguish 3 regions of human parietal cortex. Cereb Cortex 16:1418-1430.

Rushworth MF, Behrens TE, Rudebeck PH, Walton ME (2007) Contrasting roles for cingulate and orbitofrontal cortex in decisions and social behaviour. Trends Cogn Sci 11:168-176.

Sallet J, Quilodran R, Rothé M, Vezoli J, Joseph JP, Procyk E (2007) Expectations, gains, and losses in the anterior cingulate cortex. Cogn Affect Behav Neurosci 7:327-336.

Shima K, Aya K, Mushiake H, Inase M, Aizawa H, Tanji J (1991) Two movement-related foci in the primate cingulate cortex observed in signaltriggered and self-paced forelimb movements. J Neurophysiol 65:188-202.

Smith SM, Jenkinson M, Woolrich MW, Beckmann CF, Behrens TE, Johansen-Berg H, Bannister PR, De Luca M, Drobnjak I, Flitney DE, Niazy RK, Saunders J, Vickers J, Zhang Y, De Stefano N, Brady JM, Matthews PM (2004) Advances in functional and structural MR image analysis and implementation as FSL. Neuroimage 23 [Suppl 1]:S208-S219.

Spiers HJ, Maguire EA (2007) The neuroscience of remote spatial memory: a tale of two cities. Neuroscience 149:7-27.

Stephan KE, Hilgetag CC, Burns GA, O’Neill MA, Young MP, Kötter R (2000) Computational analysis of functional connectivity between areas of primate cerebral cortex. Philos Trans R Soc Lond B Biol Sci 355:111-126.

Strick PL, Dum RP, Picard N (1998) Motor areas on the medial wall of the hemisphere. Novartis Foundation Symposium 218:64-75; discussion 7580, 104-108.

Takada M, Tokuno H, Hamada I, Inase M, Ito Y, Imanishi M, Hasegawa N, Akazawa T, Hatanaka N, Nambu A (2001) Organization of inputs from cingulate motor areas to basal ganglia in macaque monkey. Eur J Neurosci 14:1633-1650.

Takada M, Nambu A, Hatanaka N, Tachibana Y, Miyachi S, Taira M, Inase M (2004) Organization of prefrontal outflow toward frontal motor-related areas in macaque monkeys. Eur J Neurosci 19:3328-3342.

Tanaka SC, Doya K, Okada G, Ueda K, Okamoto Y, Yamawaki S (2004) Prediction of immediate and future rewards differentially recruits cortico-basal ganglia loops. Nat Neurosci 7:887-893.

Tomassini V, Jbabdi S, Klein JC, Behrens TE, Pozzilli C, Matthews PM, Rushworth MF, Johansen-Berg H (2007) Diffusion-weighted imaging tractography-based parcellation of the human lateral premotor cortex identifies dorsal and ventral subregions with anatomical and functional specializations. J Neurosci 27:10259-10269.

Tomlin D, Kayali MA, King-Casas B, Anen C, Camerer CF, Quartz SR, Montague PR (2006) Agent-specific responses in the cingulate cortex during economic exchanges. Science 312:1047-1050.

Van Hoesen GW, Morecraft RJ, Vogt BA (1993) Connections of the monkey cingulate cortex. In: Neurobiology of cingulate cortex and limbic thalamus (Vogt BA, Gabriel M, eds). Boston: Birkhauser.

Vogt B (2008) Architecture, cytology and comparative organization of primate cingulate cortex. In: Cingulate neurobiology and disease (Vogt B, ed). Oxford: Oxford UP.

Vogt BA (2005) Pain and emotion interactions in subregions of the cingulate gyrus. Nat Rev Neurosci 6:533-544.

Vogt BA, Pandya DN (1987) Cingulate cortex of the rhesus monkey. II. Cortical afferents. J Comp Neurol 262:271-289.

Vogt BA, Pandya DN, Rosene DL (1987) Cingulate cortex of the rhesus monkey. I. Cytoarchitecture and thalamic afferents. J Comp Neurol 262:256-270. 
Vogt BA, Finch DM, Olson CR (1992) Functional heterogeneity in cingulate cortex: the anterior executive and posterior evaluative regions. Cerebral Cortex 2:435-443.

Vogt BA, Nimchinsky EA, Vogt LJ, Hof PR (1995) Human cingulate cortex: surface features, flat maps, and cytoarchitecture. J Comp Neurol 359:490-506.

Vogt BA, Vogt L, Farber NB, Bush G (2005) Architecture and neurocytology of monkey cingulate gyrus. J Comp Neurol 485:218-239.

Wahl M, Lauterbach-Soon B, Hattingen E, Jung P, Singer O, Volz S, Klein JC, Steinmetz H, Ziemann U (2007) Human motor corpus callosum: topography, somatotopy, and link between microstructure and function. J Neurosci 27:12132-12138.

Walton ME, Devlin JT, Rushworth MFS (2004) Interactions between decision making and performance monitoring within prefrontal cortex. Nat Neurosci 7:1259-1265.
Wang Y, Shima K, Sawamura H, Tanji J (2001) Spatial distribution of cingulate cells projecting to the primary, supplementary, and presupplementary motor areas: a retrograde multiple labelling study in the macaque monkey. Neurosci Res 39:39-49.

Wang Y, Matsuzaka Y, Shima K, Tanji J (2004) Cingulate cortical cells projecting to monkey frontal eye field and primary motor cortex. Neuroreport 15:1559-1563.

Zhang Y, Brady M, Smith S (2001) Segmentation of brain MR images through a hidden Markov random field model and the expectationmaximization algorithm. IEEE Trans Med Imaging 20:45-57.

Zilles K, Schlaug G, Matelli M, Luppino G, Schleicher A, Qü M, Dabringhaus A, Seitz R, Roland PE (1995) Mapping of human and macaque sensorimotor areas by integrating architectonic, transmitter receptor, MRI and PET data. J Anat 187:515-537. 